\title{
Interaction between meteorological parameters and COVID-19: an ecological study on 406 authorities of the UK
}

\author{
Mohammad Sarmadi $^{1,2}$ (D) Sajjad Rahimi ${ }^{1,2} \cdot$ Darrick Evensen $^{3} \cdot$ Vahid Kazemi Moghaddam $^{4}$
}

Received: 27 April 2021 / Accepted: 29 June 2021 / Published online: 9 July 2021

(C) The Author(s), under exclusive licence to Springer-Verlag GmbH Germany, part of Springer Nature 2021

\begin{abstract}
Understanding the factors affecting COVID-19 transmission is critical in assessing and mitigating the spread of the pandemic. This study investigated the transmissibility and death distribution of COVID-19 and its association with meteorological parameters to study the propagation pattern of COVID-19 in UK regions. We used the reported case and death per capita rate (as of November 13, 2020; before mass vaccination) and long-term meteorological data (temperature, humidity, precipitation, wind speed, and visibility) in 406 UK local authority levels based on publicity available secondary data. We performed correlation and regression analysis between COVID-19 variables and meteorological parameters to find the association between COVID-19 and independent variables. Student's T and Mann-Whitney's tests were used to analyze data. The correlation and regression analyses revealed that temperature, dew point, wind speed, and humidity were the most important factors associated with spread and death of COVID-19 ( $\mathrm{P}<0.05$ ). COVID-19 cases negatively correlated with humidity in areas with high population density, but the inverse in low population density areas. Wind speeds in low visibility areas, which are considered polluted air, may increase the spread of disease $(\mathrm{r}=0.42, \mathrm{P}<0.05)$ and decrease the spread in high visibility areas $(\mathrm{r}=-0.16, \mathrm{P}<0.05)$. Among low $\left(\mathrm{T}<10^{\circ} \mathrm{C}\right)$ and high $\left(\mathrm{T}>10^{\circ} \mathrm{C}\right)$ temperature areas, the average incidence rates were 2056.86 (95\% confidence interval (CI): 1909.49-2204.23) and 1446.76 (95\% CI: 1296.71-1596.81). Also, COVID-19 death per capita rates were 81.55 (95\% CI: 77.40-85.70) and 69.78 (95\% CI: 64.39-75.16) respectively. According to the comprehensive analysis, the spread of disease will be suppressed as the weather warms and humidity and wind speed decrease. Different environmental conditions can increase or decrease spread of the disease due to affecting spread of disease vectors and by altering people's behavior.
\end{abstract}

Keywords COVID-19 $\cdot$ Environmental factors $\cdot$ Meteorological parameters $\cdot$ Temperature $\cdot$ Wind speed $\cdot$ Humidity

$\overline{\text { Mohammad Sarmadi, Sajjad Rahimi, and Darrick Evensen have equal }}$ contributions.

Responsible Editor: Lotfi Aleya

Mohammad Sarmadi

msarmadi2@gmail.com

$\triangle$ Darrick Evensen

darrick.evensen@ed.ac.uk

1 Department of Environmental Health Engineering, School of Health, Torbat Heydariyeh University of Medical Sciences, Torbat Heydariyeh, Iran

2 Health Sciences Research Center, Torbat Heydariyeh University of Medical Sciences, Torbat Heydariyeh, Iran

3 Department of Politics and International Relations, University of Edinburgh, Edinburgh EH89LF, UK

4 Department of Environmental Health Engineering, Neyshabur University of Medical Sciences, Neyshabur, Iran

\section{Introduction}

The outbreak of the coronavirus disease 2019 (COVID-19) emerged in the city of Wuhan, Hubei Province, China, in December 2019, and then swiftly spread around the world within several months (Chen et al. 2020; Rubin et al. 2020). Before this pandemic, the Middle East respiratory syndrome coronavirus (MERS-CoV) and severe acute respiratory syndrome coronavirus (SARS-CoV) had brought tensions to societies but in low pressure (Wu et al. 2020a). The COVID-19 pandemic has affected a total number of $110,763,898$ persons globally, involving more than 220 countries and territories and resulted in a cumulative of 2,455,331 deaths worldwide as of 21 February 2021 (WHO 2021). During the pandemic, remarkable research was widely conducted on the factors affecting the distribution of incidence and death of COVID-19 worldwide. Despite the multitude of possible reasons proposed to explain COVID-19 dispersion, our current knowledge of this pandemic is still 
incomplete. Environmental and genetic factors have been proposed as the disease's plausible risk factors (Abdelzaher et al. 2020; X. Liu et al. 2021). Betacoronaviruses, such as MERS and SARS, are not thought to be seasonal but it is not clear for COVID-19 (Sajadi et al. 2020). In this regard, the role of environmental factors such as weather parameters (temperature, humidity, precipitation, wind speed) is still controversial among researchers. Several studies revealed a significant negative association between temperature (Biktasheva 2020; Li et al. 2020a, b, c, d; Poirier et al. 2020; Prata et al. 2020; Qi et al. 2020; Rendana 2020; Rosario et al. 2020; Rubin et al. 2020; Sehra et al. 2020; Sobral et al. 2020; Y. Wu et al. 2020a, b), humidity (Meo et al. 2020; Monami et al. 2020; Qi et al. 2020; Wu et al. 2020a, b), precipitation (Del Rio and Camacho-Ortiz 2020; Hossain et al. 2020; Menebo 2020), and wind speed (BolañoOrtiz et al. 2020; Coccia 2020; Haque and Rahman 2020; Hossain et al. 2020; Rendana 2020; Rosario et al. 2020) and incidence (or death) of COVID-19, but some studies have shown a positive correlation for temperature, humidity, and wind speed (Azuma et al. 2020; Bolaño-Ortiz et al. 2020; Kolluru et al. 2021; Luo et al. 2020; Menebo 2020; Roy 2020; Runkle et al. 2020; Singh et al. 2020; Xie and Zhu 2020). Wu et al. $(2020 \mathrm{a}, \mathrm{b})$ conducted a multivariate analysis study to investigate the effect of different weather factors such as daily average temperature, relative humidity, and wind speed on new cases and deaths of COVID-19 in China (Wu et al. $2020 \mathrm{a}, \mathrm{b}$ ). Strong correlations were found with the average temperature, humidity, and wind speed and total number of confirmed cases. The study further revealed that a $1{ }^{\circ} \mathrm{C}$ increase in temperature can reduce $3.08 \%$ (95\% CI: $1.53 \%, 4.63 \%$ ) and $1.19 \%$ (95\% CI: $0.44 \%, 1.95 \%$ ) of daily new cases and daily new deaths, respectively (Wu et al. 2020a, b). Other studies have suggested that the long-term effects of different weather parameters need to be investigated in more detail (Runkle et al. 2020; Sehra et al. 2020).

Some studies use the number of confirmed cases in relation to environmental factors, which would not be a good criterion for comparison between different regions, due to differences in testing capacity. The proportion of cases and deaths per capita population may be a better measure for comparison between regions. Also, if the areas to be considered are smaller scale or micro-regions, the results will be more accurate, because on a small scale, the climate and COVID-19 variables can be more precise for a specific geographic context, and as a result, the relationship between them is more indicative of genuine local conditions than averaged values over a large geospatial area would be (Sobral et al. 2020). Also, there is a limited study to investigate long-term effects of meteorological parameters such as temperature, wind speed, and humidity on COVID-19 distribution. Another limitation of countrylevel ecological studies is that they exclude variations in meteorological attributes at a regional level (Wu et al. 2020a, b); this generalization to the whole country can result in bias.
The United Kingdom (UK) is one of the leading European countries in the free dissemination of COVID-19 data. The UK is seventh worldwide in the number of cases $(1,557,011$ persons) and fifth in the number of deaths ( 56,533 persons) as of 26 November 2020 (GOV.UK 2021). In this study, we use multivariate regression analyses and control for the effect of population density (number of people per area of land) and median age variables, to explore the effects of weather parameters such as temperate, relative humidity, wind speed, precipitation, and dew point on the incidence of COVID-19 case and death rate at $406 \mathrm{UK}$ authorities before mass vaccination. Our results provide useful implications for researchers, medical centers, policymakers, and the public.

\section{Material and methods}

\section{Study area}

The current ecological study includes 406 local authorities that cover the majority of the UK $\left(60.85^{\circ}\right.$ to $49.95^{\circ}$ latitude and $1.76^{\circ}$ to $-8.18^{\circ}$ longitude) with approximately 248,532 square kilometers $\left(\mathrm{km}^{2}\right)$. Figure 1 illustrates the locations of the UK areas. The UK consists of four devolved nations, including England, Scotland, Wales, and Northern Ireland. In general, the climate of the UK is a temperate oceanic climate (or $\mathrm{Cfb}$ on the Köppen climate classification) — cool and often cloudy and rainy (Peel et al. 2007).

The mean temperature, rainfall, wind speed, and relative humidity for the UK from 1981 to 2010 are illustrated in Fig. 2, based on data from the UK Government Met Office (https://www.metoffice.gov.uk/) (Met Office 2021). Maximum and minimum temperature and rainfall reported $12.40\left({ }^{\circ} \mathrm{C}\right), 5.30\left({ }^{\circ} \mathrm{C}\right)$, and $1154(\mathrm{~mm})$ for the UK, respectively. The UK's population in 2020 was $67,886,011$ (83. $2 \%$ urban), with a median age of 40.5 years in 2020 (www. worldometers.info).

\section{Meteorological and weather data}

We collected the secondary data on the weather and climate parameters that were provided by several public-available websites (freemeteo.co.uk 2020; timeanddate 2020; Weather Atlas 2020). The websites report data on temperature (high, low, mean) $\left({ }^{\circ} \mathrm{C}\right)$, precipitation $(\mathrm{mm})$, relative humidity $(\%)$, dew point $\left({ }^{\circ} \mathrm{C}\right)$, wind speed $(\mathrm{km} / \mathrm{h})$, pressure (mbar), and visibility $(\mathrm{km})$ during 1985-2015. Before the statistical analysis, we conducted several data quality controls to confirm the validity of meteorological data. First, if there were data non-normally distributed in the records, they were removed from the analysis. Second, if the missing rate of data for the amount of annual data in the stations was more than $5 \%$, we decided that we would 
Fig. 1 Study location in the UK (https://www.gov.uk/); UTLA is

7-day rolling rate of new cases

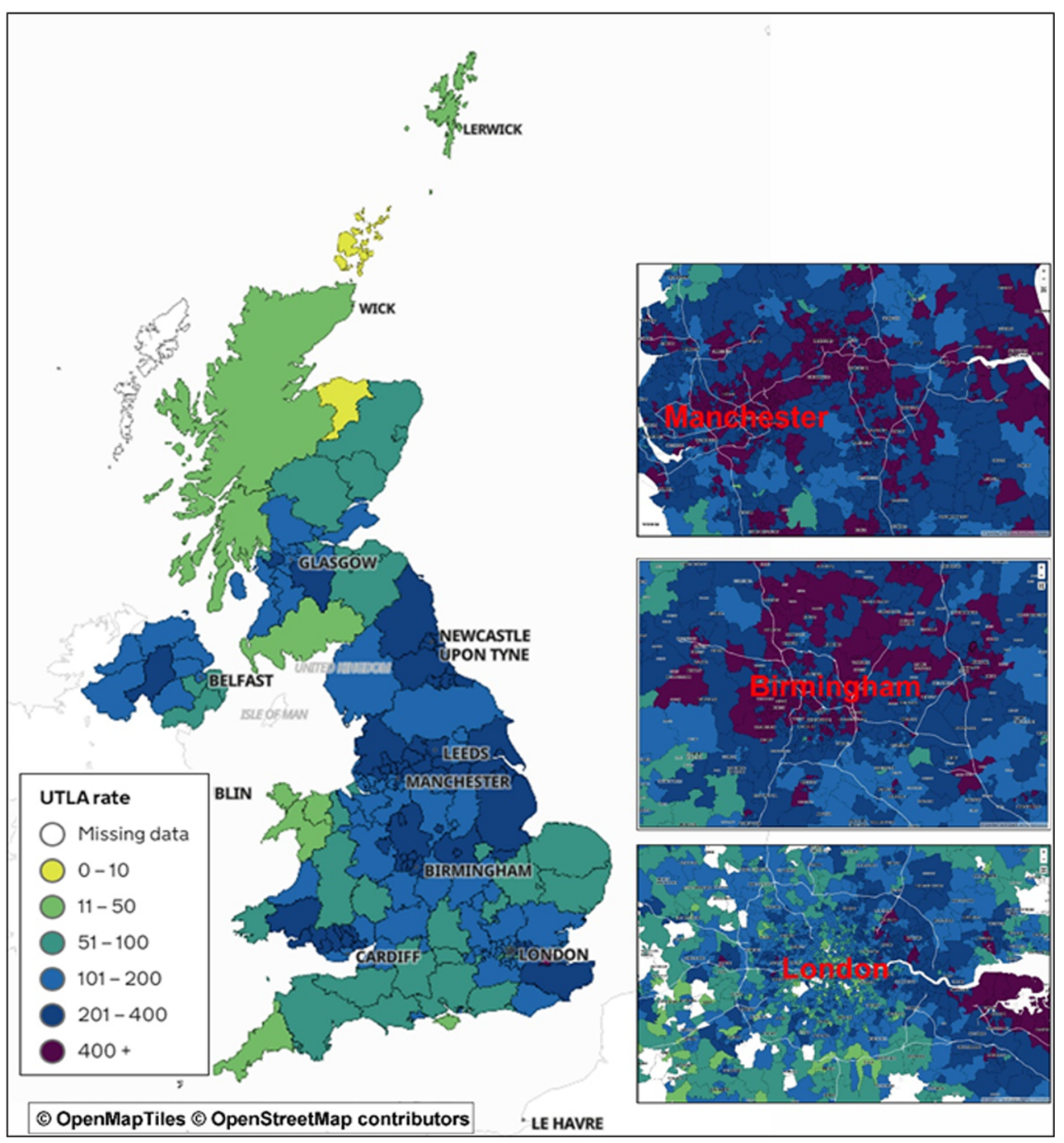

delete that station. Nevertheless, none of the stations needed to be deleted. Finally, the final data were checked with the values of meteorological parameters at nearby stations.

\section{COVID-19 and confounder variable data}

This study was carried out based on publicity available secondary data on COVID-19, which the UK Government has collected and published on its websites. Total positive confirmed cases, total deaths within 28 days of positive test, and case and death rates per 100,000 population were collected from the "official UK Government website for data and insights on COVID-19" (https:/coronavirus.data.gov.uk) as of November 13, 2020. The reported COVID-19 data are regularly updated on standard website on daily basis. Two main variables, population density (person per square area) and median age, were included in the study as potential confounding covariates (https://www.ons.gov.uk/ and https://ginform. local.gov.uk/).

\section{Statistical analysis}

Descriptive analyses were performed for all obtained data. Spearman and Pearson correlation test was applied to analyze the association between COVID-19 variables (number and rate) and demographic and meteorological parameters. Also to determine a net effect of the independent variables, multivariable regression analysis was used to explore the associations between COVID-19 case and death rates per 100,000 and meteorological factors by adjusted to population density and median age. COVID-19 data and meteorological or demographic variables were normalized before we entered them into the model, if they displayed non-normal distribution. We fixed the distributions of all studied variables with a logarithmic transformation. For comparison of mean quantitative variables, the Student T test or Mann-Whitney U test was performed. Statistical analyses were carried out using IBM SPSS Statistics 20 software and the graphical representations were performed using GraphPad Prism (version 6). All tests were 


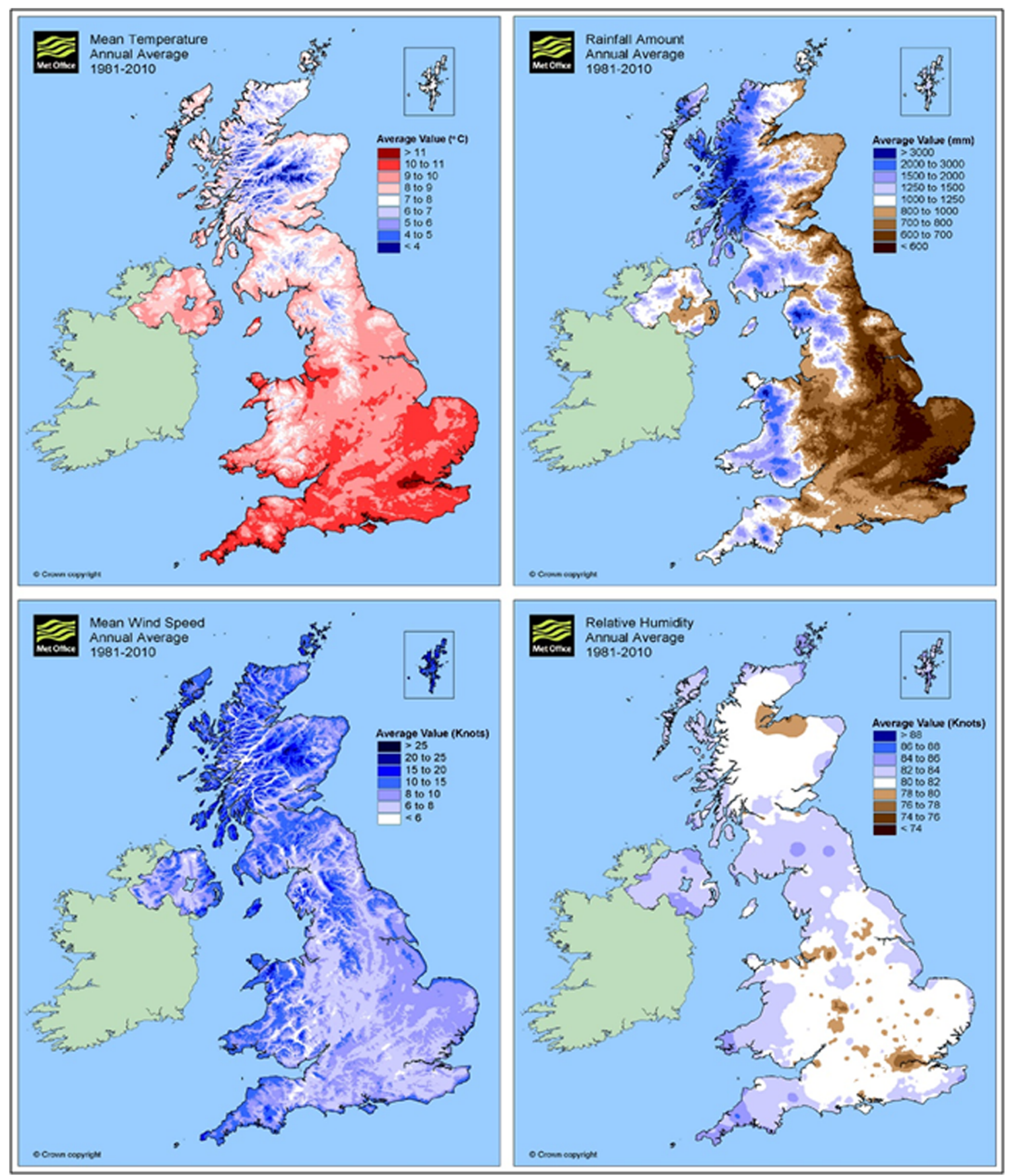

Fig. 2 Contour plots created from UK Met Office monitors showing average of meteorological parameters (Met Office 2021)

two-sided, and a value $\mathrm{P}<0.05$ was considered statistically significant.

\section{Results}

\section{Description analysis}

A total of 1,379,388 cumulative positive confirmed cases and 52,502 deaths had been reported for UK as of November 13,
2020. Lancashire, Birmingham, Leeds, and Manchester were the four regions with the most cases of COVID-19 (absolute number); also, Essex, Lancashire, Kent, and Birmingham had the most deaths of COVID-19. Blackburn with Darwen, Oldham, Manchester, and Rochdale had the highest case rate per 100,000 population, and Tameside, Wigan, Rochdale, and Wyre had the highest death rate per 100,000 population in the UK regions.

The geographic distribution (Fig. 3) for the 7-day rolling rate of new cases of COVID-19 by specimen date across 
various areas was visualized on a weekly basis from March 21 to November 13, 2020. Over the past few months (May to Aug.), the incidence of the disease has decreased but has risen again (after September to now).

The descriptive statistics for long-term meteorological parameters, COVID-19 variables (as of November 13, 2020), population density, and median age are demonstrated in Table 1.

\section{Correlation analysis}

Table 2 shows the results of correlation analysis between independent (meteorological parameters), dependent (COVID19 variables), and demographic (population density and median age) variables for the UK authorities. The analysis revealed a significant negative correlation between average high temperature $(r=-.33$ for incidence and $r=-.19$ for death), mean temperature $(r=-.22$ for incidence and $r=-.13$ for death), dew point $(r=-.29$ for incidence and $r=-.29$ for death), and COVID-19 incidence and death rates, respectively. Interestingly, the median age (also age $>65$ and $>85$ ) was inversely correlated to the COVID-19 variables, and the regions with high age $0-15$ had a significantly higher death and incidence of COVID-19 rate $(\mathrm{P}<.001)$. On the other hand, Pearson and Spearman correlation analyses were used to explore the precise associations between COVID-19 variables and meteorological parameters in various subgroups (Table 3 ). The results show that, in areas with a higher temperature $\left(>10^{\circ} \mathrm{C}\right)$, precipitation was associated with increased incidence ( $\mathrm{r}=.31)$ of COVID-19. By sharp contrast, in colder areas $\left(\leq 10{ }^{\circ} \mathrm{C}\right)$, it was linked with decreased rates of disease (incidence $(\mathrm{r}=-.18)$ and mortality $(\mathrm{r}=-.16)$ of COVID-19 $(\mathrm{P}$ $<.05)$ ).

Wind speed was significantly associated with increased $(\mathrm{P}<.05)$ incidence of COVID-19 in areas with high case rate (higher 1500 per 100,000 population), high death rate (higher 100 per 100,000 population), high humidity $(>75 \%)$, low precipitation $(\leq 48 \mathrm{~mm}$ per month), low mean temperature $\left(\leq 10^{\circ} \mathrm{C}\right)$, and low visibility $(\leq 15 \mathrm{~km})$.

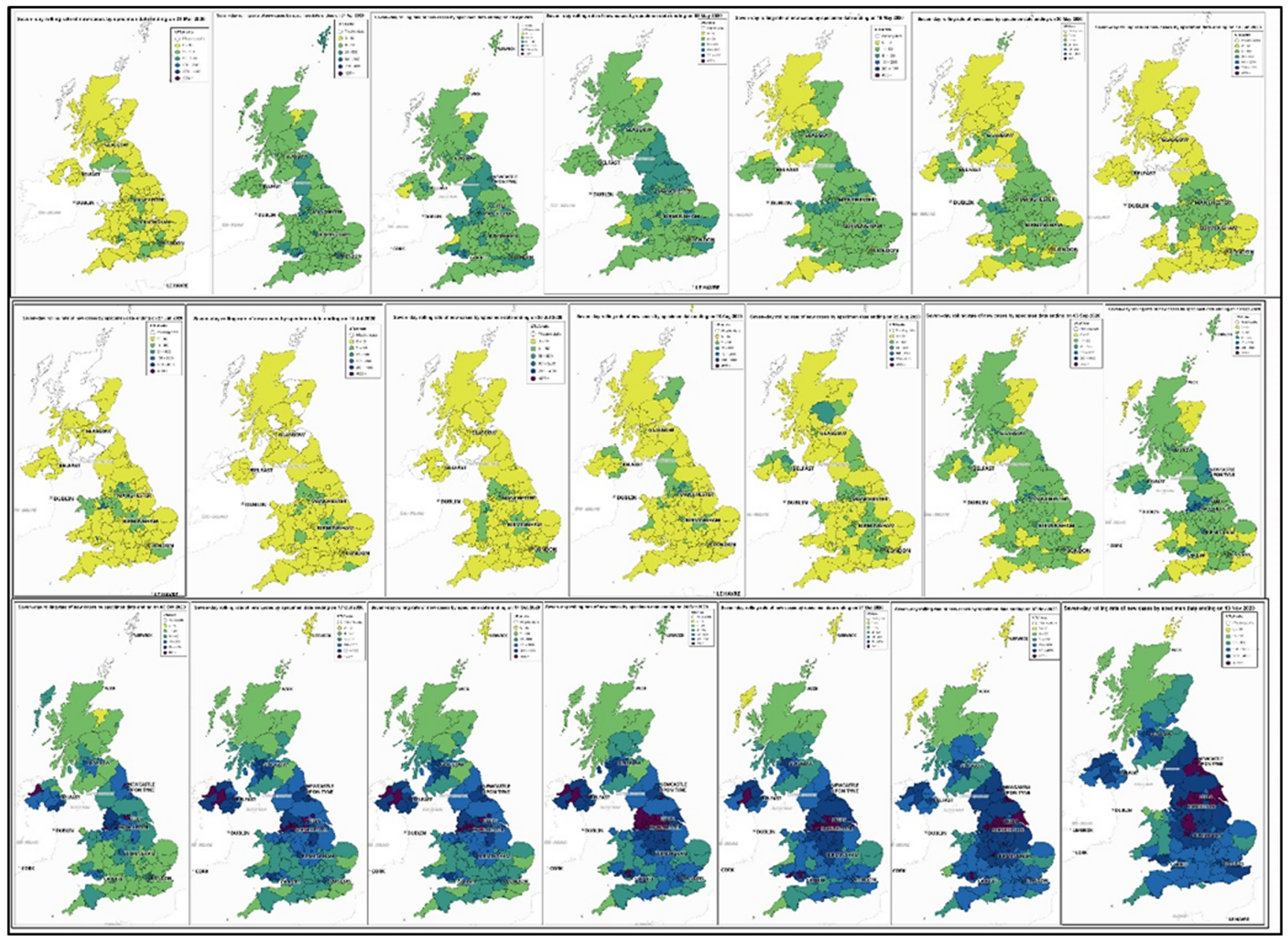

Fig. 3 Weekly distribution of COVID-19 7-day new cases rate up to 13 December 2020 for UK, each figure corresponds to a week, the colors are white $=$ missing data, yellow $=0-10$ new case rate, light green $=11-50$ new case rate, dark green $=51-100$ new case rate, light blue $=101-200$ new case rate, dark blue $=201-400$ new case rate, lollipop $=>400$ new case rate 
Table 1 Descriptive statistic of study

\begin{tabular}{|c|c|c|c|c|c|c|c|}
\hline \multirow{2}{*}{$\begin{array}{l}\text { Cases } \\
\text { Cases per 100,000 } \\
\text { population }\end{array}$} & \multirow{2}{*}{$\begin{array}{l}\text { Mean } \\
1803.78\end{array}$} & \multirow{2}{*}{$\begin{array}{l}\text { SD } \\
1037.81\end{array}$} & \multirow{2}{*}{$\begin{array}{l}\text { Median } \\
1494.00\end{array}$} & \multicolumn{2}{|c|}{$\mathrm{IQR}_{25 \%, 75 \%}$} & \multirow{2}{*}{$\begin{array}{l}\text { Min } \\
157.20\end{array}$} & \multirow{2}{*}{$\begin{array}{l}\text { Max } \\
5800.40\end{array}$} \\
\hline & & & & 1079.63 & 2276.25 & & \\
\hline $\begin{array}{l}\text { Deaths per } 100,000 \\
\text { population }\end{array}$ & 75.67 & 31.56 & 72.10 & 52.75 & 95.65 & 3.70 & 195.60 \\
\hline Average high temperate $\left({ }^{\circ} \mathrm{C}\right)$ & 21.03 & 2.00 & 21.00 & 20.00 & 22.00 & 10.00 & 34.00 \\
\hline Average low temperate $\left({ }^{\circ} \mathrm{C}\right)$ & 2.05 & 1.16 & 2.00 & 1.00 & 3.00 & -4.00 & 6.00 \\
\hline $\begin{array}{l}\text { Average mean temperate } \\
\left({ }^{\circ} \mathrm{C}\right)\end{array}$ & 10.10 & 1.09 & 10.00 & 10.00 & 11.00 & 3.00 & 19.00 \\
\hline Average precipitation (mm) & 43.04 & 20.57 & 39.25 & 30.40 & 49.70 & 2.60 & 190.00 \\
\hline $\begin{array}{l}\text { Average relative humidity } \\
(\%)\end{array}$ & 79.13 & 3.58 & 80.00 & 78.00 & 81.00 & 65.00 & 89.00 \\
\hline Average dew point $\left({ }^{\circ} \mathrm{C}\right)$ & 6.79 & 0.74 & 7.00 & 6.00 & 7.00 & 2.00 & 11.00 \\
\hline Average wind speed $(\mathrm{km} / \mathrm{h})$ & 15.68 & 2.76 & 15.00 & 14.00 & 17.00 & 11.00 & 27.00 \\
\hline Average pressure $(\mathrm{kPa})$ & 101.47 & 0.19 & 101.52 & 101.43 & 101.57 & 100.03 & 101.82 \\
\hline Average visibility (km) & 12.86 & 3.61 & 11.00 & 10.00 & 15.00 & 9.00 & 28.00 \\
\hline $\begin{array}{l}\text { Population density } \\
\left.\text { (pop. } / \mathrm{km}^{2}\right)\end{array}$ & 1512.76 & 2434.44 & 526.00 & 187.00 & 1929.00 & 8.80 & 16237.00 \\
\hline Median age (year) & 42.46 & 4.97 & 42.70 & 38.90 & 46.30 & 28.90 & 54.30 \\
\hline
\end{tabular}

Additional analyses were done for area categories (high and low variables) to explore the effect of independent variables on COVID-19 incidence and death rates (Fig. 4).

There were significant differences between COVID-19 incidence in high and low mean temperature, wind speed, dew point, pressure, and humidity ( $\mathrm{p}<0.05$ ) (Fig. 4). Among lower mean temperature and lower dew point areas, the average incidence rates were 2056.86 (CI: 1909.49-2204.23) and 2281.28 (CI: 2069.05-2493.51), and death rates were 81.55 (CI: 77.40-85.70) and 88.44 (CI: 82.32-94.55) respectively

Table 2 Bivariate correlation coefficient matrix for COVID-19 variables, meteorological parameters, and demographic variables for 406 areas of the UK

\begin{tabular}{|c|c|c|c|c|c|c|c|c|c|c|c|c|}
\hline 1. Cases & 1 & 2 & 3 & 4 & 5 & 6 & 7 & 8. & 9 & 10 & 11 & 12 \\
\hline 2. $\mathrm{PC}$ & $.702 * *$ & 1.000 & & & & & & & & & & \\
\hline 3. Death & $.899 * *$ & $.516 * *$ & 1.000 & & & & & & & & & \\
\hline 4. PD & $.470 * *$ & $.713 * *$ & $.598 * *$ & 1.000 & & & & & & & & \\
\hline 5. AHT & $-.147 * *$ & $-.327 * *$ & -0.048 & $-.188 * *$ & 1.000 & & & & & & & \\
\hline 6. ALT & 0.000 & -0.056 & 0.008 & -0.038 & $.170 * *$ & 1.000 & & & & & & \\
\hline 7. AMT & -0.078 & $-.219 * *$ & -0.027 & $-.130 *$ & $.577 * *$ & $.785^{* *}$ & 1.000 & & & & & \\
\hline 8. APrec. & -0.080 & -0.094 & -0.068 & -0.086 & -0.008 & $.185^{* *}$ & $.105^{*}$ & 1.000 & & & & \\
\hline 9. ARH & -0.037 & 0.007 & -0.069 & -0.078 & $-.622 * *$ & $-.500 * *$ & $-.616^{* *}$ & $.124 *$ & 1.000 & & & \\
\hline 10. ADP & $-.171 * *$ & $-.292 * *$ & $-.160 * *$ & $-.293 * *$ & $.373 * *$ & $.473 * *$ & $.611 * *$ & $.237 * *$ & -0.063 & 1.000 & & \\
\hline 11. AWS & 0.082 & $.192 * *$ & 0.046 & $.149 * *$ & $-.498 * *$ & 0.098 & $-.128^{*}$ & -0.062 & $.313 * *$ & -0.026 & 1.000 & \\
\hline 12. AP & -0.105 & -0.073 & -0.046 & 0.041 & $.364 * *$ & $.111 *$ & $.203 * *$ & $.219 * *$ & $-.265 * *$ & -0.002 & 0.022 & 1.000 \\
\hline 13. Visibility & $-.153 * *$ & -0.092 & $-.140 * *$ & -0.055 & $-.113^{*}$ & $-.556 * *$ & $-.463 * *$ & $.234 * *$ & $.351 * *$ & $-.189 * *$ & $-.140 * *$ & -0.032 \\
\hline 14. Median age & $-.586^{* *}$ & $-.303 * *$ & $-.507 * *$ & -.100 & $-.159 * *$ & $-.136^{* *}$ & $-.143 * *$ & .037 & $.251^{* *}$ & .011 & $.104 *$ & $.231 * *$ \\
\hline 15. Pop. density & $.429 * *$ & $.361 * *$ & $.393 * *$ & $.287 * *$ & $.261 * *$ & $.184 * *$ & $.246^{* *}$ & $-.118^{*}$ & $-.336 * *$ & .020 & $-.138 * *$ & -.052 \\
\hline 16. Age $=0-15$ & $.319 * *$ & $.229 * *$ & $.357 * *$ & $.257 * *$ & $.365 * *$ & .097 & $.204 * *$ & -.059 & $-.328 * *$ & -.009 & -.103 & .106 \\
\hline 17. Age $>$ p65 & $-.520 * *$ & $-.339 * *$ & $-.434 * *$ & $-.123^{*}$ & $-.253 * *$ & $-.129 *$ & $-.160 * *$ & .030 & $.330 * *$ & .099 & $.125^{*}$ & $.187 * *$ \\
\hline 18. Age $>85$ & $-.539 * *$ & $-.429 * *$ & $-.424 * *$ & $-.186^{* *}$ & -.073 & -.049 & -.014 & .012 & $.192 * *$ & $.186^{* *}$ & .013 & $.261 * *$ \\
\hline
\end{tabular}

** $\mathrm{P}<0.01, * \mathrm{P}<0.05, P C$ proportion of case per million, $P D$ proportion of death per million, $A H T$ average high temperature, $A L T$ average low temperature, $A M T$ average mean temperature, $A P r e c$. average precipitation, $A R H$ average relative humidity, $A D P$ average dew point, $A W S$ average wind speed, $A P$ average pressure, $P D e n c i t y$ population density (person per square kilometer) 
Table 3 Correlation of COVID-19 incidence and death (cumulative number and rate per 100,000) and meteorological parameters for 406 areas of the UK; categories for all variables ( $>$ : high and $\leq$ : low)

\begin{tabular}{|c|c|c|c|c|c|c|c|c|}
\hline Variables & Cases & $\mathrm{PC}$ & Death & PD & Cases & $\mathrm{PC}$ & death & PD \\
\hline & \multicolumn{4}{|c|}{ PC $>1500$ per 100,000} & \multicolumn{4}{|c|}{$P C \leq 1500$ per 100,000} \\
\hline Average high temperate $\left({ }^{\circ} \mathrm{C}\right)$ & -0.078 & $-.323 * *$ & -0.060 & $-.244 * *$ & $.244 * *$ & $.305 * *$ & $.287^{* *}$ & $.354 * *$ \\
\hline Average low temperate $\left({ }^{\circ} \mathrm{C}\right)$ & 0.048 & 0.021 & 0.072 & 0.101 & 0.068 & 0.110 & 0.067 & 0.056 \\
\hline Average mean temperate $\left({ }^{\circ} \mathrm{C}\right)$ & 0.005 & -0.121 & 0.047 & 0.042 & 0.114 & 0.126 & 0.125 & 0.100 \\
\hline Average precipitation (mm) & -0.063 & -0.120 & 0.002 & -0.003 & -0.072 & 0.075 & -0.074 & -0.025 \\
\hline Average relative humidity (\%) & -0.013 & 0.097 & -0.025 & -0.031 & $-.188^{*}$ & $-.338 * *$ & $-.193^{*}$ & $-.314 * *$ \\
\hline Average dew point $\left({ }^{\circ} \mathrm{C}\right)$ & -0.114 & $-.151^{*}$ & -0.106 & $-.153 *$ & -0.003 & $-.166^{*}$ & 0.003 & $-.150 *$ \\
\hline Average wind speed $(\mathrm{km} / \mathrm{h})$ & -0.006 & $.159 *$ & -0.011 & 0.131 & -0.090 & -0.118 & -0.094 & -0.102 \\
\hline Average pressure $(\mathrm{kPa})$ & $-.390 * *$ & $-.359 * *$ & $-.283 * *$ & -0.139 & -0.020 & $-.179 *$ & -0.006 & -0.080 \\
\hline \multirow[t]{2}{*}{ Average visibility $(\mathrm{km})$} & -0.113 & -0.048 & -0.082 & 0.071 & -0.139 & -0.046 & $-.169 *$ & $-.154^{*}$ \\
\hline & \multicolumn{4}{|c|}{ PD $>100$ per 100,000} & \multicolumn{4}{|c|}{$P D \leq 100$ per 100,000} \\
\hline Average high temperate $\left({ }^{\circ} \mathrm{C}\right)$ & -0.142 & $-.313 * *$ & 0.039 & -0.133 & -0.048 & $-.192 * *$ & 0.071 & -0.020 \\
\hline Average low temperate $\left({ }^{\circ} \mathrm{C}\right)$ & 0.090 & 0.143 & 0.087 & -0.002 & -0.042 & $-.139 *$ & -0.006 & -0.074 \\
\hline Average mean temperate $\left({ }^{\circ} \mathrm{C}\right)$ & -0.023 & -0.076 & 0.080 & -0.015 & -0.072 & $-.235 * *$ & -0.013 & $-.135^{*}$ \\
\hline Average precipitation (mm) & -0.096 & -0.093 & -0.054 & -0.088 & -0.061 & -0.071 & -0.072 & -0.090 \\
\hline Average relative humidity (\%) & 0.032 & 0.097 & -0.072 & 0.054 & -0.053 & -0.058 & -0.080 & -0.114 \\
\hline Average dew point $\left({ }^{\circ} \mathrm{C}\right)$ & -0.105 & -0.111 & -0.009 & -0.086 & $-.164 * *$ & $-.344 * *$ & -0.114 & $-.247 * *$ \\
\hline Average wind speed $(\mathrm{km} / \mathrm{h})$ & 0.117 & $.265 *$ & -0.009 & 0.106 & 0.008 & 0.092 & -0.013 & 0.081 \\
\hline Average pressure $(\mathrm{kPa})$ & $-.459 * *$ & $-.488 * *$ & $-.270 *$ & -0.238 & $-.267 * *$ & $-.419 * *$ & $-.139 *$ & $-.227 * *$ \\
\hline \multirow[t]{2}{*}{ Average visibility $(\mathrm{km})$} & -0.097 & -0.094 & -0.091 & -0.033 & $-.167 * *$ & -0.080 & $-.194 * *$ & $-.139 *$ \\
\hline & \multicolumn{4}{|c|}{ Population density $>300$ per $\mathrm{km}^{2}$} & \multicolumn{4}{|c|}{ Population density $\leq 300$ per $\mathbf{k m}^{2}$} \\
\hline Average high temperate $\left({ }^{\circ} \mathrm{C}\right)$ & $-.273 * *$ & $-.508 * *$ & $-.132 *$ & $-.308 * *$ & -.085 & $-.181 *$ & .023 & -.070 \\
\hline Average low temperate $\left({ }^{\circ} \mathrm{C}\right)$ & -.088 & $-.186^{* *}$ & -.056 & $-.134 *$ & -.035 & -.040 & -.081 & -.098 \\
\hline Average mean temperate $\left({ }^{\circ} \mathrm{C}\right)$ & $-.165 * *$ & $-.369 * *$ & -.078 & $-.214 * *$ & -.140 & $-.202 *$ & -.131 & $-.215^{*}$ \\
\hline Average precipitation (mm) & -.011 & -.062 & .008 & -.036 & -.116 & -.112 & $-.196^{*}$ & $-.205^{*}$ \\
\hline Average relative humidity (\%) & $.130 *$ & $.263 * *$ & .038 & .097 & -.071 & $-.206^{*}$ & -.039 & -.174 \\
\hline Average dew point $\left({ }^{\circ} \mathrm{C}\right)$ & $-.227 * *$ & $-.346^{* *}$ & $-.175 * *$ & $-.281 * *$ & -.094 & $-.256^{* *}$ & -.144 & $-.332 * *$ \\
\hline Average wind speed $(\mathrm{km} / \mathrm{h})$ & $.193 * *$ & $.337 * *$ & $.136^{*}$ & $.260 * *$ & -.034 & .021 & -.076 & .030 \\
\hline Average pressure $(\mathrm{kPa})$ & $-.413 * *$ & $-.473 * *$ & $-.259 * *$ & $-.245 * *$ & -.133 & $-.349 * *$ & -.078 & $-.294 * *$ \\
\hline \multirow[t]{2}{*}{ Average visibility $(\mathrm{km})$} & -.048 & .065 & -.047 & .113 & -.088 & -.087 & -.096 & -.109 \\
\hline & \multicolumn{4}{|c|}{ Median age $>45$ years } & \multicolumn{4}{|c|}{ Median age $\leq 45$ years } \\
\hline Average high temperate $\left({ }^{\circ} \mathrm{C}\right)$ & -.067 & -.105 & .022 & -.052 & $-.311 * *$ & $-.446 * *$ & $-.174 * *$ & $-.251 * *$ \\
\hline Average low temperate $\left({ }^{\circ} \mathrm{C}\right)$ & $-.197 *$ & -.158 & -.177 & -.115 & .001 & -.063 & .029 & -.022 \\
\hline Average mean temperate $\left({ }^{\circ} \mathrm{C}\right)$ & $-.269 * *$ & $-.274 * *$ & -.189 & -.173 & -.107 & $-.223 * *$ & -.038 & -.111 \\
\hline Average precipitation (mm) & -.082 & -.133 & -.146 & -.162 & -.047 & -.103 & -.008 & -.068 \\
\hline Average relative humidity (\%) & -.080 & $-.248^{*}$ & -.078 & $-.230 *$ & $.146^{*}$ & $.206 * *$ & .058 & .040 \\
\hline Average dew point $\left({ }^{\circ} \mathrm{C}\right)$ & $-.229 *$ & $-.394 * *$ & $-.202 *$ & $-.361 * *$ & $-.169 * *$ & $-.226^{* *}$ & $-.159 *$ & $-.227 * *$ \\
\hline Average wind speed $(\mathrm{km} / \mathrm{h})$ & .034 & .043 & -.030 & -.004 & $.176^{* *}$ & $.260 * *$ & $.140 *$ & $.213 * *$ \\
\hline Average pressure $(\mathrm{kPa})$ & $-.250 *$ & $-.429 * *$ & -.166 & $-.320 * *$ & $-.303 * *$ & $-.341 * *$ & $-.184 *$ & $-.184 *$ \\
\hline \multirow[t]{2}{*}{ Average visibility $(\mathrm{km})$} & -.081 & -.098 & -.084 & -.075 & -.073 & -.042 & -.071 & -.015 \\
\hline & \multicolumn{4}{|c|}{ Percentage of children $>20 \%$} & \multicolumn{4}{|c|}{ Percentage of children $\leq 20$} \\
\hline Average high temperate $\left({ }^{\circ} \mathrm{C}\right)$ & $-.352 * *$ & $-.494 * *$ & -.169 & $-.352 * *$ & $-.196^{* *}$ & $-.258 * *$ & $-.139 *$ & $-.178^{* *}$ \\
\hline Average low temperate $\left({ }^{\circ} \mathrm{C}\right)$ & -.026 & -.100 & .017 & -.025 & -.063 & -.064 & -.086 & -.090 \\
\hline Average mean temperate $\left({ }^{\circ} \mathrm{C}\right)$ & -.159 & $-.286 * *$ & -.002 & -.090 & $-.167 * *$ & $-.208 * *$ & $-.163^{*}$ & $-.188^{* *}$ \\
\hline Average precipitation (mm) & -.175 & $-.244 *$ & -.100 & -.171 & -.062 & -.076 & -.075 & -.084 \\
\hline Average relative humidity (\%) & .159 & $.271 * *$ & .058 & .175 & .026 & -.047 & .032 & -.086 \\
\hline Average dew point $\left({ }^{\circ} \mathrm{C}\right)$ & $-.310 * *$ & $-.393 * *$ & $-.225^{*}$ & $-.243 *$ & $-.169 * *$ & $-.259 * *$ & $-.206 * *$ & $-.312 * *$ \\
\hline Average wind speed $(\mathrm{km} / \mathrm{h})$ & $.265^{*}$ & $.418^{* *}$ & .144 & $.331 * *$ & .071 & .109 & .042 & .078 \\
\hline
\end{tabular}


Table 3 (continued)

\begin{tabular}{|c|c|c|c|c|c|c|c|c|}
\hline Variables & Cases & $\mathrm{PC}$ & Death & $\mathrm{PD}$ & Cases & $\mathrm{PC}$ & death & $\mathrm{PD}$ \\
\hline Average pressure $(\mathrm{kPa})$ & $-.335^{* *}$ & $-.418 * *$ & $-.263 *$ & $-.433 * *$ & $-.359 * *$ & $-.377 * *$ & $-.248 * *$ & $-.190 * *$ \\
\hline \multirow[t]{2}{*}{ Average visibility (km) } & -.028 & .045 & -.037 & .050 & $-.136^{*}$ & $-.128 *$ & -.113 & -.063 \\
\hline & \multicolumn{4}{|c|}{ Mean temperature $>10^{\circ} \mathrm{C}$} & \multicolumn{4}{|c|}{ Mean temperature $\leq 10^{\circ} \mathrm{C}$} \\
\hline Average high temperate $\left({ }^{\circ} \mathrm{C}\right)$ & 0.069 & 0.033 & $.214^{*}$ & $.251 * *$ & $-.144 *$ & $-.272 * *$ & -0.110 & $-.257 * *$ \\
\hline Average low temperate $\left({ }^{\circ} \mathrm{C}\right)$ & -0.019 & 0.083 & -0.057 & -0.012 & $.215^{* *}$ & $.298 * *$ & $.171 *$ & $.236 * *$ \\
\hline Average mean temperate $\left({ }^{\circ} \mathrm{C}\right)$ & 0.157 & -0.012 & $.183^{*}$ & 0.059 & 0.114 & $.138^{*}$ & 0.094 & 0.127 \\
\hline Average precipitation (mm) & $.194 *$ & $.313^{* *}$ & 0.157 & 0.148 & $-.161 *$ & $-.181 * *$ & $-.154 *$ & $-.155^{*}$ \\
\hline Average relative humidity (\%) & -0.059 & -0.073 & $-.177 *$ & $-.275^{* *}$ & $-.127 *$ & $-.244 * *$ & -0.029 & $-.190 * *$ \\
\hline Average dew point $\left({ }^{\circ} \mathrm{C}\right)$ & $-.319 * *$ & $-.328 * *$ & $-.370 * *$ & $-.369 * *$ & -0.046 & -0.126 & -0.058 & $-.187 * *$ \\
\hline Average wind speed $(\mathrm{km} / \mathrm{h})$ & 0.099 & 0.115 & 0.068 & 0.025 & 0.015 & $.137 *$ & -0.022 & 0.104 \\
\hline Average pressure $(\mathrm{kPa})$ & -0.166 & $-.192 *$ & -0.101 & -0.100 & $-.364 * *$ & $-.387 * *$ & $-.304 * *$ & $-.306^{* *}$ \\
\hline \multirow[t]{2}{*}{ Average visibility $(\mathrm{km})$} & -0.118 & -0.108 & -0.055 & 0.026 & $-.249 * *$ & $-.270 * *$ & $-.226^{* *}$ & $-.207 * *$ \\
\hline & \multicolumn{4}{|c|}{ Mean precipitation $>48 \mathrm{~mm}$} & \multicolumn{4}{|c|}{ Mean precipitation $\leq 48 \mathrm{~mm}$} \\
\hline Average high temperate $\left({ }^{\circ} \mathrm{C}\right)$ & 0.013 & $-.216^{* *}$ & 0.094 & -0.090 & $-.273 * *$ & $-.441 * *$ & $-.156^{*}$ & $-.279 * *$ \\
\hline Average low temperate $\left({ }^{\circ} \mathrm{C}\right)$ & -0.030 & $-.236 * *$ & 0.033 & -0.130 & 0.047 & 0.110 & -0.017 & 0.045 \\
\hline Average mean temperate $\left({ }^{\circ} \mathrm{C}\right)$ & 0.079 & $-.195^{*}$ & 0.129 & -0.117 & $-.157^{*}$ & $-.210^{* *}$ & -0.131 & -0.130 \\
\hline Average precipitation (mm) & 0.017 & 0.146 & -0.043 & 0.034 & -0.073 & -0.094 & -0.111 & $-.167^{*}$ \\
\hline Average relative humidity $(\%)$ & -0.019 & 0.134 & -0.078 & 0.015 & -0.066 & $-.134 *$ & -0.037 & $-.173 *$ \\
\hline Average dew point $\left({ }^{\circ} \mathrm{C}\right)$ & -0.012 & -0.158 & -0.044 & $-.239 * *$ & $-.263 * *$ & $-.363^{* *}$ & $-.237 * *$ & $-.335^{* *}$ \\
\hline Average wind speed $(\mathrm{km} / \mathrm{h})$ & 0.096 & 0.087 & 0.095 & 0.080 & 0.059 & $.228 * *$ & 0.017 & $.178 * *$ \\
\hline Average pressure $(\mathrm{kPa})$ & -0.136 & $-.346^{* *}$ & -0.100 & $-.305^{* *}$ & $-.453 * *$ & $-.484 * *$ & $-.298 * *$ & $-.262 * *$ \\
\hline \multirow[t]{2}{*}{ Average visibility (km) } & $-.194 *$ & -0.025 & $-.193 *$ & 0.000 & -0.115 & -0.105 & -0.096 & -0.075 \\
\hline & \multicolumn{4}{|c|}{ Mean relative humidity $>75 \%$} & \multicolumn{4}{|c|}{ Mean relative humidity $\leq \mathbf{7 5 \%}$} \\
\hline Average high temperate $\left({ }^{\circ} \mathrm{C}\right)$ & $-.153 * *$ & $-.288 * *$ & -0.096 & $-.221 * *$ & 0.187 & $.255^{*}$ & 0.208 & 0.172 \\
\hline Average low temperate $\left({ }^{\circ} \mathrm{C}\right)$ & 0.079 & 0.084 & 0.041 & 0.031 & $-.241 *$ & -0.212 & $-.247 *$ & -0.179 \\
\hline Average mean temperate $\left({ }^{\circ} \mathrm{C}\right)$ & -0.045 & -0.110 & -0.047 & -0.099 & 0.225 & -0.033 & 0.220 & 0.009 \\
\hline Average precipitation (mm) & -0.077 & -0.062 & -0.102 & -0.098 & $.308^{* *}$ & $.292 *$ & $.293 *$ & 0.175 \\
\hline Average relative humidity (\%) & $-.153 * *$ & $-.202 * *$ & -0.115 & $-.197 * *$ & $.271 *$ & 0.162 & 0.181 & 0.013 \\
\hline Average dew point $\left({ }^{\circ} \mathrm{C}\right)$ & $-.172 * *$ & $-.272 * *$ & $-.184 * *$ & $-.310 * *$ & -0.097 & -0.073 & 0.037 & 0.201 \\
\hline Average wind speed $(\mathrm{km} / \mathrm{h})$ & 0.041 & $.124^{*}$ & 0.029 & $.120 *$ & 0.223 & 0.124 & 0.208 & 0.128 \\
\hline Average pressure $(\mathrm{kPa})$ & $-.416^{* *}$ & $-.444 * *$ & $-.327 * *$ & $-.309 * *$ & 0.103 & 0.028 & 0.022 & $-.261 *$ \\
\hline \multirow[t]{2}{*}{ Average visibility (km) } & $-.205^{* *}$ & $-.165 * *$ & $-.174 * *$ & -0.088 & 0.178 & $.256^{*}$ & 0.208 & 0.179 \\
\hline & \multicolumn{4}{|c|}{ Mean dew point $>6^{\circ} \mathrm{C}$} & \multicolumn{4}{|c|}{ Mean dew point $\leq 6^{\circ} \mathrm{C}$} \\
\hline Average high temperate $\left({ }^{\circ} \mathrm{C}\right)$ & -0.115 & $-.310 * *$ & 0.077 & -0.007 & 0.081 & $.191 *$ & -0.035 & 0.042 \\
\hline Average low temperate $\left({ }^{\circ} \mathrm{C}\right)$ & 0.009 & 0.033 & -0.005 & 0.018 & $.250 * *$ & $.353 * *$ & $.241 * *$ & $.336^{* *}$ \\
\hline Average mean temperate $\left({ }^{\circ} \mathrm{C}\right)$ & -0.061 & $-.221 * *$ & 0.018 & -0.082 & $.257 * *$ & $.455^{* *}$ & $.209^{*}$ & $.403 * *$ \\
\hline Average precipitation (mm) & 0.083 & $.153^{*}$ & 0.047 & 0.062 & $-.220 *$ & $-.363 * *$ & -0.149 & $-.235^{*}$ \\
\hline Average relative humidity (\%) & -0.077 & -0.019 & $-.165^{*}$ & $-.208 * *$ & -0.058 & $-.206^{*}$ & 0.045 & -0.093 \\
\hline Average dew point $\left({ }^{\circ} \mathrm{C}\right)$ & $-.228 * *$ & $-.201 * *$ & $-.273 * *$ & $-.308 * *$ & $.309 * *$ & $.370 * *$ & $.315^{* *}$ & $.360 * *$ \\
\hline Average wind speed $(\mathrm{km} / \mathrm{h})$ & $.129 *$ & $.236^{* *}$ & 0.083 & $.176^{* *}$ & -0.157 & -0.172 & $-.184 *$ & $-.219^{*}$ \\
\hline Average pressure $(\mathrm{kPa})$ & $-.353 * *$ & $-.462 * *$ & $-.177^{*}$ & $-.295 * *$ & $-.262 * *$ & -0.078 & $-.293 * *$ & 0.038 \\
\hline \multirow[t]{2}{*}{ Average visibility (km) } & $-.130 *$ & -0.030 & $-.134 *$ & -0.025 & $-.258 * *$ & $-.373 * *$ & $-.220 *$ & $-.233^{*}$ \\
\hline & \multicolumn{4}{|c|}{ Mean wind speed $>15 \mathrm{~km} / \mathrm{h}$} & \multicolumn{4}{|c|}{ Mean wind speed $\leq 15 \mathrm{~km} / \mathrm{h}$} \\
\hline Average high temperate $\left({ }^{\circ} \mathrm{C}\right)$ & -0.036 & -0.128 & 0.045 & -0.059 & $-.164 *$ & $-.372 * *$ & -0.090 & $-.239 * *$ \\
\hline Average low temperate $\left({ }^{\circ} \mathrm{C}\right)$ & 0.105 & 0.139 & 0.070 & 0.118 & -0.080 & $-.239 * *$ & -0.023 & -0.119 \\
\hline Average mean temperate $\left({ }^{\circ} \mathrm{C}\right)$ & 0.098 & 0.064 & 0.118 & 0.110 & $-.164 *$ & $-.384 * *$ & -0.109 & $-.249 * *$ \\
\hline Average precipitation (mm) & -0.056 & -0.152 & -0.004 & -0.064 & -0.062 & 0.010 & -0.082 & -0.029 \\
\hline Average relative humidity (\%) & $-.202 *$ & $-.295 * *$ & -0.163 & $-.326^{* *}$ & 0.049 & $.148 *$ & -0.021 & 0.025 \\
\hline Average dew point $\left({ }^{\circ} \mathrm{C}\right)$ & -0.097 & -0.138 & -0.042 & -0.085 & $-.241 * *$ & $-.455^{* *}$ & $-.247 * *$ & $-.445 * *$ \\
\hline
\end{tabular}


Table 3 (continued)

\begin{tabular}{|c|c|c|c|c|c|c|c|c|}
\hline Variables & Cases & $\mathrm{PC}$ & Death & $\mathrm{PD}$ & Cases & $\mathrm{PC}$ & death & $\mathrm{PD}$ \\
\hline Average wind speed $(\mathrm{km} / \mathrm{h})$ & -0.049 & 0.062 & -0.062 & 0.119 & $.191 * *$ & $.211 * *$ & $.193 * *$ & $.257 * *$ \\
\hline Average pressure $(\mathrm{kPa})$ & $-.400 * *$ & $-.378 * *$ & $-.265 * *$ & $-.218 *$ & $-.269 * *$ & $-.425 * *$ & $-.181 *$ & $-.325 * *$ \\
\hline \multirow[t]{2}{*}{ Average visibility (km) } & $-.337 * *$ & $-.310 * *$ & $-.263 * *$ & -0.119 & 0.034 & $.199 * *$ & -0.049 & 0.037 \\
\hline & \multicolumn{4}{|c|}{ Mean pressure $>101.40 \mathrm{KPa}$} & \multicolumn{4}{|c|}{ Mean pressure $\leq 101.40 \mathrm{kPa}$} \\
\hline Average high temperate $\left({ }^{\circ} \mathrm{C}\right)$ & -0.061 & $-.273 * *$ & 0.121 & -0.115 & -0.008 & -0.079 & -0.075 & -0.105 \\
\hline Average low temperate $\left({ }^{\circ} \mathrm{C}\right)$ & 0.002 & -0.062 & 0.033 & -0.020 & 0.115 & 0.096 & 0.025 & 0.007 \\
\hline Average mean temperate $\left({ }^{\circ} \mathrm{C}\right)$ & -0.094 & $-.242 * *$ & 0.015 & -0.107 & 0.112 & 0.050 & -0.003 & -0.049 \\
\hline Average precipitation (mm) & -0.018 & -0.085 & -0.003 & -0.084 & -0.197 & -0.222 & -0.198 & -0.112 \\
\hline Average relative humidity $(\%)$ & -0.077 & -0.038 & $-.143 *$ & $-.138^{*}$ & -0.117 & -0.109 & -0.012 & -0.016 \\
\hline Average dew point $\left({ }^{\circ} \mathrm{C}\right)$ & $-.160 *$ & $-.326^{* *}$ & -0.118 & $-.323 * *$ & 0.118 & 0.027 & 0.028 & -0.086 \\
\hline Average wind speed $(\mathrm{km} / \mathrm{h})$ & 0.061 & $.203 * *$ & -0.011 & $.148^{*}$ & -0.071 & -0.005 & -0.003 & 0.095 \\
\hline Average pressure $(\mathrm{kPa})$ & $-.204 * *$ & $-.444 * *$ & -0.095 & $-.381 * *$ & $-.565 * *$ & $-.668 * *$ & $-.519 * *$ & $-.506 * *$ \\
\hline \multirow[t]{2}{*}{ Average visibility $(\mathrm{km})$} & -0.112 & -0.079 & -0.101 & -0.068 & $-.293 *$ & -0.220 & $-.284 *$ & -0.182 \\
\hline & \multicolumn{4}{|c|}{ Mean visibility $>15 \mathrm{~km}$} & \multicolumn{4}{|c|}{ Mean visibility $\leq 15 \mathrm{~km}$} \\
\hline Average high temperate $\left({ }^{\circ} \mathrm{C}\right)$ & -0.074 & -0.096 & -0.103 & -0.143 & $-.184 * *$ & $-.493 * *$ & -0.023 & $-.232 * *$ \\
\hline Average low temperate $\left({ }^{\circ} \mathrm{C}\right)$ & 0.065 & 0.009 & 0.122 & 0.089 & $-.175 * *$ & $-.228 * *$ & $-.185 * *$ & $-.230 * *$ \\
\hline Average mean temperate $\left({ }^{\circ} \mathrm{C}\right)$ & 0.036 & -0.001 & 0.078 & 0.040 & $-.220 * *$ & $-.511 * *$ & $-.153^{*}$ & $-.370 * *$ \\
\hline Average precipitation (mm) & -0.123 & -0.118 & -0.089 & -0.052 & -0.035 & $-.133 *$ & -0.004 & -0.108 \\
\hline Average relative humidity (\%) & -0.123 & $-.181 *$ & -0.051 & -0.073 & 0.084 & $.207 * *$ & -0.005 & 0.004 \\
\hline Average dew point $\left({ }^{\circ} \mathrm{C}\right)$ & -0.125 & $-.217 * *$ & -0.084 & $-.172 *$ & $-.243 * *$ & $-.396^{* *}$ & $-.250 * *$ & $-.437 * *$ \\
\hline Average wind speed $(\mathrm{km} / \mathrm{h})$ & -0.117 & $-.161^{*}$ & -0.027 & -0.014 & $.188 * *$ & $.419 * *$ & 0.069 & $.225 * *$ \\
\hline Average pressure $(\mathrm{kPa})$ & $-.285^{* *}$ & $-.382 * *$ & $-.186^{*}$ & $-.210 *$ & $-.387 * *$ & $-.490 * *$ & $-.239 * *$ & $-.365 * *$ \\
\hline Average visibility (km) & $-.206^{* *}$ & -0.133 & $-.192 *$ & -0.096 & -0.128 & -0.075 & -0.111 & 0.006 \\
\hline
\end{tabular}

$* * \mathrm{P}<0.01, * \mathrm{P}<0.05, P C$ proportion of case per million, $P D$ proportion of death per million

(Fig. 4). Also, COVID-19 incidence in higher mean temperature and dew point areas were 1446.76 (CI: 1296.71-1596.81) and 1605.98 (CI: 1484.40-1727.56 (Fig. 4). However, the regions with low mean temperature $\left(\leq 10^{\circ} \mathrm{C}\right)$, low pressure $(\leq 101.40 \mathrm{kPa})$, and low dew point $\left(\leq 6^{\circ} \mathrm{C}\right)$ showed significantly higher COVID-19 incidence when compared to high-value regions (Fig. 4).

\section{Regression analysis}

Table 4 presents the results of regression analyses of COVID19 incidence and death rates and meteorological parameters. Results revealed a negative association of high and mean temperature, dew point, and pressure (adjusted for population density and median age) on COVID-19 incidence rate ( $\mathrm{P}$ $<0.001)$. On the other hand, there were positive significant associations for humidity and wind speed and COVID-19 incidence rate.

In multiple regression analysis, the results showed that relative humidity, wind speed, and population density have positive associations with COVID-19 incidence rate $(\mathrm{P}<0.05$, Table 5). In the case of COVID-19 death rate (adjusted for population density and median age), significant associations were found for high temperature, mean temperature, dew point, wind speed, and pressure $(\mathrm{p}<0.01)$.

\section{Discussion}

The novel coronavirus pandemic has become a major challenge globally, with approximately 2.5 million deaths and as of yet no clear exit from the pandemic (WHO 2021). The pattern of COVID-19 distribution is related to extra-human conditions, such as weather and demographic structural conditions (Cohen 2020). Several factors could affect the incidence and death distribution of COVID-19 in various regions. However, moving toward the micro-regional (or local authority level) studies can further clarify the effects of environmental factors such as meteorological parameters (Sobral et al. 2020). In this analysis of 406 UK local authorities, change in temperature (mean, high and low), wind speed, humidity, precipitation, dew point, pressure, visibility, population density, and median age were associated with the cumulative number and rate of incidence and death of COVID-19. The primary correlation results of this study (Table 2) indicate that, of these several meteorological factors, long-term high temperature and dew point have been the most 


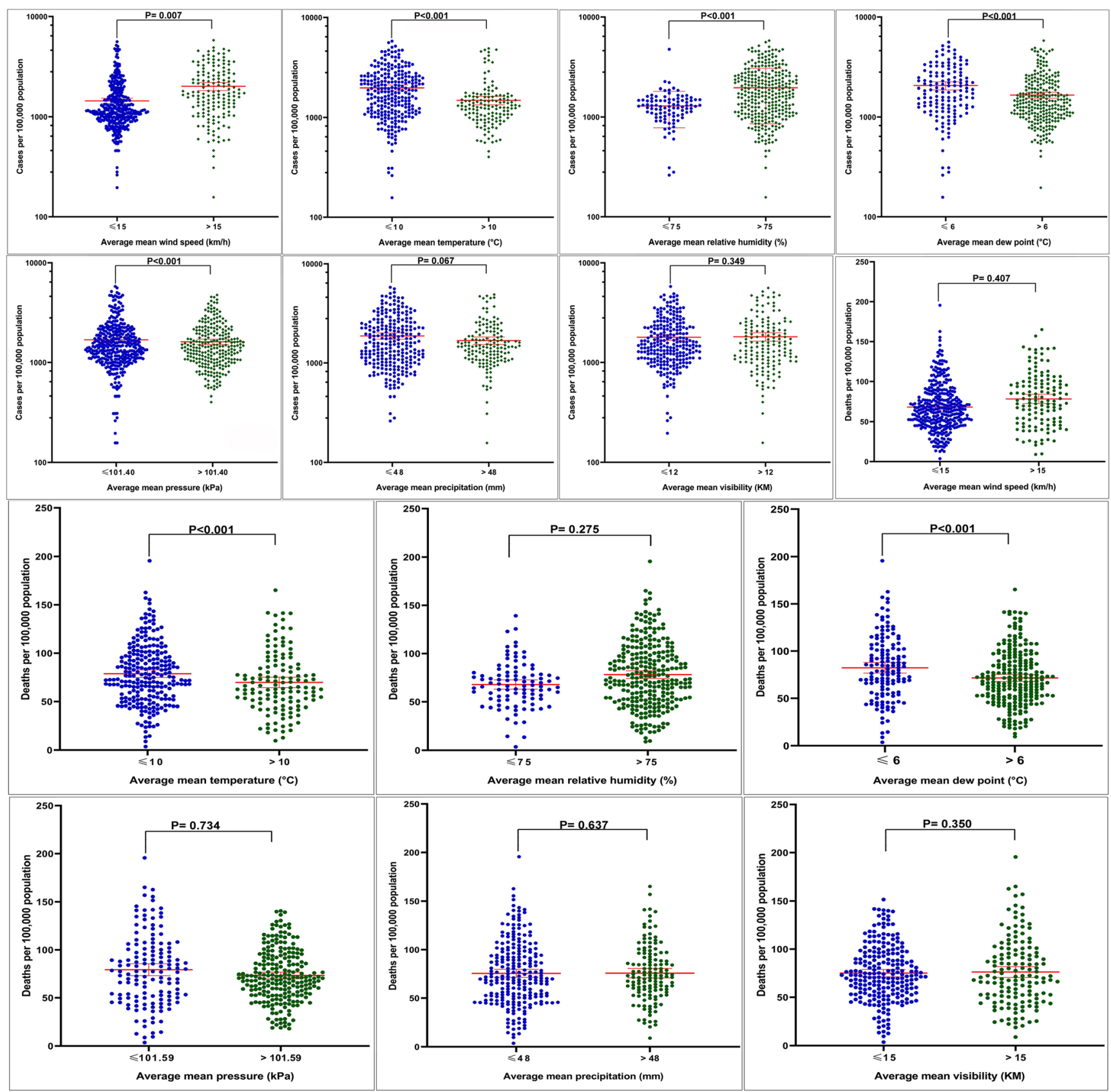

Fig. 4 Differences of COVID-19 variables in low and high subgroups by two independent samples test. Red horizontal lines represent group means with $95 \%$ CI. P is $P$-value

significant in reducing COVID-19 incidence and death rates, but wind speed significantly increased those rates. It should be noted that the effect of environmental parameters on disease incidence can be more reliable than death, because before the dire situation, their people live in natural environments and everyone's conditions are the same, but for mortality, other parameters such as the number of hospital beds, ICU bed, and the health care system of regions can play a more effective role. There are several studies indicating that regions with higher temperatures have lower rates of the disease (Biktasheva 2020; Li et al. 2020a, b, c, d; Poirier et al. 2020; Prata et al. 2020; Qi et al. 2020; Rendana 2020;
Rosario et al. 2020; Rubin et al. 2020; Sehra et al. 2020; Sobral et al. 2020; Y. Wu et al. 2020). Our study also revealed a significant association of the COVID-19 incidence and death rate with average high temperature and mean temperature in the multivariable analysis, after adjusting for confounding variables.

\section{Temperature and COVID-19}

There are some factors like latitude and socio-demographic factors (Bao et al. 2016) and air pollution (Cai et al. 2007) that influence the association between temperature and mortality. 
Table 4 Regression coefficients for mutually adjusted associations between COVID-19 incidence and death per capita rates and meteorological parameters (adjusted with population density and median age)

\begin{tabular}{|c|c|c|c|c|c|c|c|}
\hline \multirow[t]{2}{*}{ Covariates } & \multicolumn{2}{|c|}{$\begin{array}{l}\text { Unstandardized } \\
\text { coefficients }\end{array}$} & \multirow{2}{*}{$\begin{array}{l}\text { Standardized } \\
\text { coefficients } \\
\text { Beta }\end{array}$} & \multirow[t]{2}{*}{$\mathrm{t}$} & \multirow[t]{2}{*}{ Sig. } & \multirow[t]{2}{*}{ VIF } & \multirow[t]{2}{*}{$\mathrm{R}^{2}$} \\
\hline & $\mathrm{B}$ & Std. error & & & & & \\
\hline \multicolumn{8}{|l|}{ Log cases per million } \\
\hline $\begin{array}{l}\text { Log average high } \\
\text { temperate }\end{array}$ & -1.382 & .280 & -.247 & -4.933 & .000 & 1.109 & .236 \\
\hline $\begin{array}{l}\text { Log average low } \\
\text { temperate }\end{array}$ & -.085 & .062 & -.072 & -1.377 & .169 & 1.047 & .188 \\
\hline $\begin{array}{l}\text { Log average mean } \\
\text { temperate }\end{array}$ & -.677 & .240 & -.145 & -2.823 & .005 & 1.125 & .200 \\
\hline $\begin{array}{l}\text { Log average } \\
\text { precipitation }\end{array}$ & -.079 & .058 & -.067 & -1.349 & .178 & 1.021 & .180 \\
\hline $\begin{array}{l}\text { Log average relative } \\
\text { humidity }\end{array}$ & 2.658 & .624 & .220 & 4.262 & .000 & 1.147 & .218 \\
\hline Log average dew point & -.918 & .222 & -.204 & -4.137 & .000 & 1.061 & .221 \\
\hline Log average wind speed & .518 & .164 & .157 & 3.167 & .002 & 1.044 & .205 \\
\hline Log average pressure & -80.487 & 16.775 & -.254 & -4.798 & .000 & 1.105 & .250 \\
\hline Log average visibility & -.036 & .118 & -.016 & -.304 & .761 & 1.121 & .183 \\
\hline \multicolumn{8}{|l|}{ Deaths per million } \\
\hline $\begin{array}{l}\text { Log average high } \\
\text { temperate }\end{array}$ & -132.660 & 39.743 & -.182 & -3.338 & .001 & 1.093 & .135 \\
\hline $\begin{array}{l}\text { Log average low } \\
\text { temperate }\end{array}$ & -13.267 & 9.177 & -.083 & -1.446 & .149 & 1.057 & .105 \\
\hline $\begin{array}{l}\text { Log average mean } \\
\text { temperate }\end{array}$ & -82.902 & 33.807 & -.138 & -2.452 & .015 & 1.136 & .121 \\
\hline $\begin{array}{l}\text { Log average } \\
\text { precipitation }\end{array}$ & -10.774 & 8.517 & -.067 & -1.265 & .207 & 1.006 & .107 \\
\hline $\begin{array}{l}\text { Log average relative } \\
\text { humidity }\end{array}$ & 92.310 & 90.375 & .058 & 1.021 & .308 & 1.131 & .105 \\
\hline Log average dew point & -165.964 & 31.885 & -.276 & -5.205 & .000 & 1.081 & .175 \\
\hline Log average wind speed & 78.067 & 24.272 & .171 & 3.216 & .001 & 1.037 & .133 \\
\hline Log average pressure & -9487.608 & 2227.484 & -.247 & -4.259 & .000 & 1.100 & .154 \\
\hline Log average visibility & 11.802 & 16.832 & .039 & .701 & .484 & 1.105 & .106 \\
\hline
\end{tabular}

Bold numbers are statistically significant, VIF variance inflation factor
In fact, people who live far from or near the equator have a relatively strong adaptive capacity to cold and heat, respectively (Sobral et al. 2020). On the other hand, low temperature may increase the transmissibility of viruses, either by "conserving virulence or weakening the host by cooling the body or drying the respiratory tract" (Jaakkola et al. 2014). Temperature is a multifaceted variable with differential effects on the spread of the virus under different conditions. For example in a study published in JAMA, it was reported that at temperatures below $11^{\circ} \mathrm{C}$, the incidence and mortality rate decreases with increasing average temperature, but the relationship reverses above $11^{\circ} \mathrm{C}$, which is in line with our results (Rubin et al. 2020). This finding can be related to more human activities or behaviors in the warm region like the use of parks, total attendance in the park, exercising, playing, and getting fresh air (Eliasson et al. 2007; Thorsson et al. 2007). Also, de Montigny et al. (2012) conducted a study (2007-2008) and found that a $14 \%$ and $23 \%$ increase in pedestrians was linked with a $5{ }^{\circ} \mathrm{C}$ increase in outdoor ambient temperature and a shift from snowy to dry condition, respectively. The SARSCoV-2 is primarily and secondary transmitted among people through respiratory droplets, contact routes, and close contact with patients during travels (Adedokun 2020; Burke 2020; Chan et al. 2020; Li et al. 2020a, b, c, d; Liu et al. 2020; Magurano et al. 2020). The findings of a study stated that increased person-to-person contact can increase transmission of the virus on warm and/or sunny days (Azuma et al. 2020). Although with increasing temperature, the transmissibility of COVID-19 disease may decrease due to the reduced survival time of the virus on the surfaces (Magurano et al. 2020; Riddell et al. 2020); however, these findings should be interpreted cautiously, because in the absence of mitigation programs such as travel and outdoor activities restrictions, school closures, isolation, and quarantine of patients, there would be no significant reduction in incidence rates. Therefore, temperature should be considered with the other 
Table 5 Regression coefficients for mutually adjusted associations between COVID-19 incidence and death per capita rates and the most important factors (adjusted together)

\begin{tabular}{|c|c|c|c|c|c|c|c|}
\hline \multirow[t]{2}{*}{ Covariates } & \multicolumn{2}{|c|}{ Unstandardized coefficients } & \multirow{2}{*}{$\begin{array}{l}\text { Standardized coefficients } \\
\text { Beta }\end{array}$} & \multirow[t]{2}{*}{$\mathrm{t}$} & \multirow[t]{2}{*}{ Sig. } & \multirow[t]{2}{*}{ VIF } & \multirow[t]{2}{*}{$\mathrm{R}^{2}$} \\
\hline & $\mathrm{B}$ & Std. error & & & & & \\
\hline \multicolumn{8}{|l|}{ Log cases per million } \\
\hline Log population density & .153 & .030 & .426 & 5.012 & .000 & 3.010 & \multirow[t]{8}{*}{0.284} \\
\hline Log average dew point & -1.388 & .401 & -.280 & -3.459 & .001 & 2.735 & \\
\hline Log average pressure & -51.405 & 18.150 & -.163 & -2.832 & .005 & 1.379 & \\
\hline Log average relative humidity & 2.009 & .965 & .181 & 2.082 & .038 & 3.166 & \\
\hline Log average wind speed & .447 & .184 & .143 & 2.429 & .016 & 1.451 & \\
\hline Log average mean temperate & .870 & .505 & .171 & 1.724 & .086 & 4.103 & \\
\hline Log average high temperate & .110 & .467 & .021 & .236 & .814 & 3.187 & \\
\hline Log median age & -.189 & .364 & -.044 & -.518 & .605 & 2.993 & \\
\hline \multicolumn{8}{|l|}{ Deaths per million } \\
\hline Log population density & .193 & .028 & .631 & 6.952 & .000 & 2.955 & \multirow[t]{8}{*}{0.248} \\
\hline Log median age & 1.711 & .330 & .473 & 5.180 & .000 & 2.990 & \\
\hline Log average dew point & -1.524 & .377 & -.350 & -4.038 & .000 & 2.703 & \\
\hline Log average wind speed & .568 & .173 & .202 & 3.279 & .001 & 1.368 & \\
\hline Log average pressure & -39.528 & 16.195 & -.151 & -2.441 & .015 & 1.374 & \\
\hline Log average relative humidity & -.982 & .862 & -.105 & -1.140 & .255 & 3.021 & \\
\hline Log average mean temperate & .162 & .460 & .038 & .352 & .725 & 4.176 & \\
\hline Log average high temperate & .692 & .421 & .154 & 1.646 & .101 & 3.148 & \\
\hline
\end{tabular}

Bold numbers are statistically significant, VIF variance inflation factor

aforementioned environmental factors, including population density, air pollution, wind speed, and humidity. On the other hand, our results revealed a significant positive association of COVID-19 incidence with humidity in the multivariable analysis. Also, it is worthy to mention that the effect sizes are consistently larger for cases per capita compared to deaths per capita. This likely makes sense, because weather has its impact on disease transmission which is one factor out of several ones effecting deaths.

\section{Humidity, dew point, and COVID-19}

The multivariable regression associations we discovered, especially within the humidity range, are consistent with the direct relationship of humidity and incidence rate or cumulative number of COVID-19 in other studies (Bolaño-Ortiz et al. 2020; Luo et al. 2020; Singh et al. 2020). Humidity has been introduced as one of the best predictors for the transmission of COVID-19 (Runkle et al. 2020). Several studies found that higher humidity was correlated with increased transmission of viruses such as influenza through fomites, even if viral aerosol transmission reduces in high temperature (Ijaz et al. 1985; Lowen et al. 2007). Runkle et al. (2020) using a lag nonlinear model and case-crossover study showed exposure to humidity was positively associated with transmission of COVID-19 in
US cities (Runkle et al. 2020). In our study, it was also shown that in areas with low wind speeds, higher humidity can increase the incidence of disease $(r=.15, \mathrm{P}<.05)$. This may be due to the increased concentration of virus in exposure environment, especially in densest regions, and resulting in the inoculum effect (Rubin et al. 2020), both for COVID-19 and other viruses (Chu et al. 2004; Poulsen et al. 2002; Virlogeux et al. 2015; Watanabe et al. 2010). Correlation and regression analyses showed that the dew point is one of the most important parameters in the COVID-19 distribution (Tables 3, 4, and 5). Higher dew points occur in warmer environments where the persistence of the virus on fomite is shorter (Biryukov et al. 2020; Magurano et al. 2020; Riddell et al. 2020). Category analysis in our study showed at higher dew points $\left(>6^{\circ} \mathrm{C}\right.$ ), the association between COVID-19 variables and the dew point was negative, but at lower dew points $(\leq 6$ ${ }^{\circ} \mathrm{C}$ ), it was positive.

\section{Wind speed and COVID-19}

Based on our overall results, there is also a positive significant association between wind speed and COVID-19 incidence and death rates, especially in densest regions, which may be related to the inoculum effect. SARS-CoV-2 can spread more in windy conditions because it increases air circulation 
(Coşkun et al. 2021; Rosario et al. 2020). However, our study here investigates the interaction between wind speed and COVID-19 variables in the UK regions under different COVID-19 and environmental subgroups (high and low categories). Interestingly, a positive correlation was observed between the incidence of COVID-19 and wind speed in areas with high death rates, high humidity, high population density, high percent of children, low temperature, low visibility, and low precipitation (Table 3). Also, in areas with low wind speed, there is a positive correlation between wind speed and incidence of COVID-19 ( $P>0.05)$. In line with these results, Coccia (2020) shows that the regions with little wind and high levels of air pollution have higher cases of COVID19 (Coccia 2020). The studies also revealed that wind speed has an inverse significant relationship with the rate of COVID-19 (Ahmadi et al. 2020; Rosario et al. 2020) and viral load of SARS (Jiang et al. 2003). On the other hand, among the meteorological variables, wind speed and humidity were the main factors that influenced visibility (these factors more related on particle matter) (Majewski et al. 2014; Zhao et al. 2013). Also, in spring, when there is more rainfall and the humidity is higher, low visibility has been reported (Du et al. 2013). Based on our results, higher humidity correlated with a lower temperature (mean, max, min) (Table 2). Therefore, due to the reasons stated above, wind speed, dew point, and humidity are the most important effective meteorological parameters in the SARS-CoV-2 transmission, as revealed in multivariable regression analysis (Tables 4 and 5). Feng et al. (2020) stated that relative humidity and wind speed may cause SARS-CoV-2 heavily loaded droplets to travel long distances in the air (Feng et al. 2020). Data assessing interactions between humidity, wind speed, and visibility (particulate matter) for SARS-CoV-2 are essential to confirm this claim at the local authority level. Gong et al. (2015) assert that severe fever with thrombocytopenia syndrome virus could be transmitted by aerosol transmission as a possible transmission route (Gong et al. 2015), which must also be considered in the case of COVID-19.

\section{Precipitation and COVID-19}

Higher total numbers and per capita rates of COVID-19 cases and deaths are not correlated with a lower mean precipitation. Another interesting result of this study is the direct association between mean precipitation and COVID-19 incidence at high temperatures, but it has an inverse correlation in low temperate ( $\mathrm{P}<0.05)$. This can be related to doing outdoor activities at high temperatures while at low temperatures people usually stay home and reduce close contact and thus reduce the spread of the virus. In line with our results, Sobral et al. (2020) found that precipitation has a positive correlation and SARS-CoV-2 transmission (Sobral et al. 2020).

\section{Density population and COVID-19}

Overall, there is a tendency that both incidence and death per capita rates increase with population density. The primary mechanism for the associations of population density on COVID-19 is probably associated with increased droplet and potentially airborne transmission in close contact (Bahl et al. 2020; Van Doremalen et al. 2020). We find that density has affected the timing of the outbreak in each county, with denser locations more likely to have an early outbreak. Several studies mention that population density is a dominant factor influencing the spread of SARS-CoV-2 (Bhadra et al. 2020; Rashed and Kodera 2020). We also found local authorities with high percentages of children (age between 0 and 15) had higher cases and death per capita rate of COVID-19. The study conducted by Dattner et al. (2021) revealed children had milder symptoms than adults when infected, which may have increased cases of COVID-19 (Dattner et al. 2021). This could be one of the reasons for the direct correlation between the percentage of children and increase in the disease.

\section{Strengths and limitations}

To the best of our knowledge, this is one of the most comprehensive studies of meteorological parameters on the incidence and death of COVID-19 at the local authority level. To date, scientific research of COVID-19 outcomes has been conducted at the country level, state level, and city level. There is a substantial difference between our current study and the previous research in the sense that this study examined all local authorities in the UK, whereas the other studies mostly employ data from select cities and are generalized for all of a country, especially in the US and in European countries (Bolaño-Ortiz et al. 2020; Coccia 2020; Coşkun et al. 2021; Gupta et al. 2020; Magnusson et al. 2020; Y. Wu et al. 2020). These types of studies have provided more detailed information to advance plausible predictions for incidence and death (cumulative and per capita rate), to recognize potential gaps in public health services such as hospital facilities, and to change or modify decision makers' perceptions toward environmental factors affecting the disease. Our local authority level analysis has allowed us to more holistically explore relevant contributions of median age, population density, and meteorological parameter changes on a given region's incidence and deaths associated with COVID-19. A strong innovation of our study is the ability to compare across the range of covariates for interaction effects - where the association with incidence and death rates runs in different directions at different levels of the variable. These findings can help to inform policy makers on the risk of COVID-19 transmission in different areas of a country. Further studies should be conducted, given that the COVID-19 variables will continue to change. 
Despite the strengths of our study, there are several potential limitations in ecological studies which are known as "ecological fallacy." In these studies, researchers used variables that report the mean characteristics of groups in place of individual-level outcomes and are most useful in analyzing new hypotheses (Morgenstern 1995). Generalizability remains a potential concern, due to our focus on the local authority level. Lack of access to precise local meteorological data from a number of regions, which use meteorological stations data near the area, should be considered. Furthermore, out of a large number of possible factors that affect the incidence of and death from COVID-19, only meteorological were considered specific to the UK regions. However, caution should be taken in the extrapolation of the results beyond the UK region-based meteorological parameters entered in this study. Also, to determine the causal role of a variable as a risk factor, prospective studies such as longitudinal cohort should be designed, which we suggest in future research.

\section{Conclusions}

The primary results of this study reveal that wind speed, humidity, temperature, and population density may account for geographical variation in the spread of SARS-CoV-2 across the UK local authorities. It should be noted that weather parameters might have negative or positive effects on the transmission of COVID-19 in different conditions (such as warm or cold environments); however, we acknowledge that other parameters like people's behaviors or governments' regulations, quarantine schemes, and also interactions between climatic variables with each other could change the pattern of the disease. These findings may find relevant in policy decisions for prevention, control, and management of the COVID-19 pandemic, especially at the regional level throughout the countries.

\begin{abstract}
Abbreviations COVID-19, coronavirus disease 2019; SARS-CoV, severe acute respiratory syndrome coronavirus; MERS-CoV, Middle East respiratory syndrome coronavirus; UK, United Kingdom; T, temperature; $\mathrm{PC}$, proportion of case per million; $\mathrm{PD}$, proportion of death per million

Availability of data and materials All data generated or analyzed during this study are included in this published article ("Material and methods") and publicly available dataset. Data related to the COVID-19 variables available on https://coronavirus.data.gov.uk website.
\end{abstract}

Author contribution Mohammad Sarmadi: conceptualization; methodology; data curation; formal analysis; writing — original draft preparation; writing - review and editing; supervision; funding acquisition. Darrick Evensen: methodology; formal analysis; writing — original draft preparation; writing - review and editing. Sajjad Rahimi: methodology; data curation; formal analysis; writing - original draft; writing - review and editing. Vahid Kazemi Moghaddam: conceptualization; data curation; writing - original draft preparation. All authors read and approved the manuscript.

Funding This work support by the Torbat Heydariyeh University of Medical Sciences (grant number: 99000217 and 99000007).

\section{Declarations}

Ethics approval and consent to participate Ethics codes (IR.THUMS.REC.1400.008 and IR.THUMS.REC.1399.003) were obtained from the Ethics Committee of Torbat Heydariyeh University of Medical Sciences. The consent to participate is not applicable.

Consent for publication Not applicable

Competing interests The authors declare no competing interests.

\section{References}

Abdelzaher H, Saleh BM, Ismail HA, Hafiz M, Gabal MA, Mahmoud M, Hashish S, Gawad RMA, Gharieb RY, Abdelnaser A (2020) COVID-19 Genetic and environmental risk factors: a look at the evidence. Front Pharmacol 11:579415. https://doi.org/10.3389/ fphar.2020.579415

Adedokun KA (2020) A close look at the biology of SARS-CoV-2, and the potential influence of weather conditions and seasons on COVID-19 case spread. Environ Sci Pollut Res Int 9(1):77. https:// doi.org/10.1186/s40249-020-00688-1

Ahmadi, M., Sharifi, A., Dorosti, S., Ghoushchi, S. J., \& Ghanbari, N. (2020). Investigation of effective climatology parameters on COVID-19 outbreak in Iran. Science of the Total Environment, 138705.

Weather Atlas. (2020). Weather forecast for cities. Retrieved 10 Nov, 2020, from https://www.weather-atlas.com/

Azuma K, Kagi N, Kim H, Hayashi M (2020) Impact of climate and ambient air pollution on the epidemic growth during COVID-19 outbreak in Japan. Environ Res 190:110042. https://doi.org/10. 1016/j.envres.2020.110042

Bahl, P., Doolan, C., de Silva, C., Chughtai, A. A., Bourouiba, L., \& MacIntyre, C. R. (2020). Airborne or droplet precautions for health workers treating COVID-19? The Journal of infectious diseases.

Bao J, Wang Z, Yu C, Li X (2016) The influence of temperature on mortality and its Lag effect: a study in four Chinese cities with different latitudes. BMC Public Health 16(1):375. https://doi.org/ 10.1186/s12889-016-3031-z

Bhadra A, Mukherjee A, Sarkar K (2020) Impact of population density on Covid-19 infected and mortality rate in India. Modeling Earth Systems and Environment. 7:623-629. https://doi.org/10.1007/ s40808-020-00984-7

Biktasheva IV (2020) Role of a habitat ' s air humidity in Covid-19 mortality. Sci Total Environ 736:138763. https://doi.org/10.1016/j. scitotenv.2020.138763

Biryukov, J., Boydston, J. A., Dunning, R. A., Yeager, J. J., Wood, S., Reese, A. L., ... Zeitouni, N. E. (2020). Increasing temperature and relative humidity accelerates inactivation of SARS-CoV-2 on surfaces. 5(4). doi: https://doi.org/10.1128/mSphere.00441-20

Bolaño-Ortiz TR, Camargo-Caicedo Y, Puliafito SE, Ruggeri MF, Bolaño-Diaz S, Pascual-Flores R, Saturno J, Ibarra-Espinosa S, Mayol-Bracero OL, Torres-Delgado E, Cereceda-Balic F (2020) Spread of SARS-CoV-2 through Latin America and the Caribbean region: a look from its economic conditions, climate and air 
pollution indicators. Environ Res 191:109938. https://doi.org/10. 1016/j.envres.2020.109938

Burke, R. M. (2020). Active monitoring of persons exposed to patients with confirmed COVID-19-United States, January-February 2020. MMWR. Morbidity and mortality weekly report, 69.

Cai Q-C, Lu J, Xu Q-F, Guo Q, Xu D-Z, Sun Q-W, Yang H, Zhao GM, Jiang Q-W (2007) Influence of meteorological factors and air pollution on the outbreak of severe acute respiratory syndrome. Public Health 121(4):258-265

Chan, J. F.-W., Yuan, S., Kok, K.-H., Tonn, K. K-W, Chu, H., Yang, J., ... Poon, R. W.-S. (2020). A familial cluster of pneumonia associated with the 2019 novel coronavirus indicating person-to-person transmission: a study of a family cluster. The Lancet, 395(10223), 514-523.

Chen, N., Zhou, M., Dong, X., Qu, J., Gong, F., Han, Y., ... Wei, Y. (2020). Epidemiological and clinical characteristics of 99 cases of 2019 novel coronavirus pneumonia in Wuhan, China: a descriptive study. The Lancet, 395(10223), 507-513.

Chu, C.-M., Poon, L. L. M., Cheng, V. C. C., Chan, K.-S., Hung, I. F. N., Wong, M. M. L., ... Yuen, K.-Y. (2004). Initial viral load and the outcomes of SARS. Canadian Medical Association Journal, 171(11), 1349-1352. doi: https://doi.org/10.1503/cmaj.1040398

Coccia M (2020) How do low wind speeds and high levels of air pollution support the spread of COVID-19? Atmos Pollut Res. 12:437-445. https://doi.org/10.1016/j.apr.2020.10.002

Cohen J (2020) Sick time. Science 367(6484):1294-1297. https://doi.org/ 10.1126/science.367.6484.1294

Coșkun H, Yıldırım N, Gündüz S (2021) The spread of COVID-19 virus through population density and wind in Turkey cities. Sci Total Environ 751:141663. https://doi.org/10.1016/j.scitotenv.2020. 141663

Dattner I, Goldberg Y, Katriel G, Yaari R, Gal N, Miron Y, Ziv A, Sheffer R, Hamo Y, Huppert A (2021) The role of children in the spread of COVID-19: using household data from Bnei Brak, Israel, to estimate the relative susceptibility and infectivity of children. PLoS computational biology 17(2):e1008559

de Montigny L, Ling R, Zacharias J (2012) The effects of weather on walking rates in nine cities. Environment and Behavior 44(6):821840

Del Rio C, Camacho-Ortiz A (2020) Will environmental changes in temperature affect the course of COVID-19? Braz. J Infect Dis 24(3): 261-263. https://doi.org/10.1016/j.bjid.2020.04.007

Du K, Mu C, Deng J, Yuan F (2013) Study on atmospheric visibility variations and the impacts of meteorological parameters using high temporal resolution data: an application of Environmental Internet of Things in China. International Journal of Sustainable Development \& World Ecology 20(3):238-247

Eliasson I, Knez I, Westerberg U, Thorsson S, Lindberg F (2007) Climate and behaviour in a Nordic city. Landscape and Urban Planning 82(1-2):72-84

Feng Y, Marchal T, Sperry T, Yi H (2020) Influence of wind and relative humidity on the social distancing effectiveness to prevent COVID19 airborne transmission: a numerical study. Journal of Aerosol Science 147:105585. https://doi.org/10.1016/j.jaerosci.2020. 105585

freemeteo.co.uk. (2020). The Weather- 29,071 weather forecasts for all cities, villages and toponyms. Retrieved 10 Nov, 2020, from https:// freemeteo.co.uk

Gong Z, Gu S, Zhang Y, Sun J, Wu X, Ling F, Shi W, Zhang P, Li D, Mao H, Zhang L, Wen D, Zhou B, Zhang H, Huang Y, Zhang R, Jiang J, Lin J, Xia S, Chen E, Chen Z (2015) Probable aerosol transmission of severe fever with thrombocytopenia syndrome virus in southeastern China. Clinical Microbiology and Infection 21(12): 1115-1120. https://doi.org/10.1016/j.cmi.2015.07.024

GOV.UK. (2021). Coronavirus (COVID-19) in the UK. 2021, from https://coronavirus.data.gov.uk/
Gupta S, Raghuwanshi GS, Chanda A (2020) Effect of weather on COVID-19 spread in the US: a prediction model for India in 2020. Sci Total Environ 728:138860. https://doi.org/10.1016/j.scitotenv. 2020.138860

Haque SE, Rahman M (2020) Association between temperature, humidity, and COVID-19 outbreaks in Bangladesh. Environ Sci Policy 114:253-255. https://doi.org/10.1016/j.envsci.2020.08.012

Hossain MS, Ahmed S, Uddin MJ (2020) Impact of weather on COVID19 transmission in south Asian countries: an application of the ARIMAX model. Sci Total Environ 143315:143315. https://doi. org/10.1016/j.scitotenv.2020.143315

Ijaz M, Brunner A, Sattar S, Nair RC, Johnson-Lussenburg C (1985) Survival characteristics of airborne human coronavirus 229E. Journal of General Virology 66(12):2743-2748

Jaakkola, K., Saukkoriipi, A., Jokelainen, J., Juvonen, R., Kauppila, J., Vainio, O., ... the, K.-S. G (2014). Decline in temperature and humidity increases the occurrence of influenza in cold climate. Environmental Health, 13(1), 22. doi: https://doi.org/10.1186/ 1476-069X-13-22

Jiang S, Huang L, Chen X, Wang J, Wu W, Yin S, Chen W, Zhan J, Yan L, Ma L, Li J, Huang Z (2003) Ventilation of wards and nosocomial outbreak of severe acute respiratory syndrome among healthcare workers. Chinese Medical Journal 116(9):1293-1297

Kolluru SSR, Patra AK, Nazneen, Shiva Nagendra SM (2021) Association of air pollution and meteorological variables with COVID-19 incidence: evidence from five megacities in India. Environmental Research 195:110854. https://doi.org/10.1016/j. envres.2021.110854

Li AY, Hannah TC, Durbin JR, Dreher N, McAuley FM, Marayati NF, Spiera Z, Ali M, Gometz A, Kostman JT, Choudhri TF (2020a) Multivariate analysis of Black race and environmental temperature on COVID-19 in the US. Am J Med Sci 360(4):348-356. https://doi. org/10.1016/j.amjms.2020.06.015

Li H, Xu XL, Dai DW, Huang ZY, Ma Z, Guan YJ (2020b) Air pollution and temperature are associated with increased COVID-19 incidence: a time series study. Int J Infect Dis 97:278-282. https://doi.org/10. 1016/j.ijid.2020.05.076

Li, Q., Guan, X., Wu, P., Wang, X., Zhou, L., Tong, Y., ... Wong, J. Y. (2020c). Early transmission dynamics in Wuhan, China, of novel coronavirus-infected pneumonia. New England Journal of Medicine., 382, 1199, 1207

Li, W., Zhang, B., Lu, J., Liu, S., Chang, Z., Cao, P., .. Tao, K. (2020d). The characteristics of household transmission of COVID-19. Clinical Infectious Diseases.

Liu, J., Liao, X., Qian, S., Yuan, J., Wang, F., Liu, Y., ... Zhang, Z. (2020). Community transmission of severe acute respiratory syndrome coronavirus 2, Shenzhen, China, 2020.

Liu X, Huang J, Li C, Zhao Y, Wang D, Huang Z, Yang K (2021) The role of seasonality in the spread of COVID-19 pandemic. Environmental Research 195:110874. https://doi.org/10.1016/j. envres.2021.110874

Lowen AC, Mubareka S, Steel J, Palese P (2007) Influenza virus transmission is dependent on relative humidity and temperature. PLoS Pathog 3(10):e151

Luo, W., Majumder, M., Liu, D., Poirier, C., Mandl, K., Lipsitch, M., \& Santillana, M. (2020). The role of absolute humidity on transmission rates of the COVID-19 outbreak.

Magnusson W, Hsu YL, Lin HC, Wei HM, Lai HC, Hwang KP (2020) Temperature and the difference in impact of SARS CoV-2 infection (COVID-19) between tropical and non-tropical regions in Taiwan. PeerJ 36:101790. https://doi.org/10.1016/j.tmaid.2020.101790

Magurano F, Baggieri M, Marchi A, Rezza G, Nicoletti L (2020) SARS$\mathrm{CoV}-2$ infection: the environmental endurance of the virus can be influenced by the increase of temperature. Clin Microbiol Infect. 27: 289.e5-289.e7. https://doi.org/10.1016/j.cmi.2020.10.034 
Majewski, G., Czechowski, P. O., Badyda, A., \& Brandyk, A. (2014). Effect of air pollution on visibility in urban conditions. Warsaw case study. Environment Protection Engineering, 40(2).

Menebo MM (2020) Temperature and precipitation associate with Covid19 new daily cases: a correlation study between weather and Covid19 pandemic in Oslo, Norway. Sci Total Environ 737:139659. https://doi.org/10.1016/j.scitotenv.2020.139659

Meo, S. A., Abukhalaf, A. A., Alomar, A. A., Aljudi, T. W., Bajri, H. M., Sami, W., ... Hajjar, W. (2020). Impact of weather conditions on incidence and mortality of COVID-19 pandemic in Africa. Eur Rev Med Pharmacol Sci, 24(18), 9753-9759. doi: https://doi.org/10. 26355/eurrev_202009_23069

Met Office. (2021). UK climate averages. Retrieved 1 June, 2021, from https://www.metoffice.gov.uk/research/climate/maps-and-data/ukclimate-averages/gcn3y $738 \mathrm{~h}$

Monami M, Silverii A, Mannucci E (2020) Potential impact of climate on novel corona virus (COVID-19) epidemic. Transbound Emerg Dis 62(7):e371-e372. https://doi.org/10.1097/jom.0000000000001885

Morgenstern H (1995) Ecologic studies in epidemiology: concepts, principles, and methods. Annual Review of Public Health 16(1):61-81

Peel MC, Finlayson BL, McMahon TA (2007) Updated world map of the Köppen-Geiger climate classification. Hydrol. Earth Syst. Sci. 11(5):1633-1644. https://doi.org/10.5194/hess-11-1633-2007

Poirier C, Luo W, Majumder MS, Liu D, Mandl KD, Mooring TA, Santillana M (2020) The role of environmental factors on transmission rates of the COVID-19 outbreak: an initial assessment in two spatial scales. Sci Rep 10(1):17002. https://doi.org/10.1038/s41598020-74089-7

Poulsen A, Qureshi K, Lisse I, Kofoed P-E, Nielsen J, Vestergaard B, Aaby P (2002) A household study of chickenpox in Guinea-Bissau: intensity of exposure is a determinant of severity. Journal of Infection 45(4):237-242

Prata DN, Rodrigues W, Bermejo PH (2020) Temperature significantly changes COVID-19 transmission in (sub)tropical cities of Brazil. $\mathrm{Sci}$ Total Environ 729:138862. https://doi.org/10.1016/j.scitotenv.2020. 138862

Qi H, Xiao S, Shi R, Ward MP, Chen Y, Tu W, Su Q, Wang W, Wang X, Zhang Z (2020) COVID-19 transmission in Mainland China is associated with temperature and humidity: a time-series analysis. $\mathrm{Sci}$ Total Environ 728:138778. https://doi.org/10.1016/j.scitotenv.2020. 138778

Rashed, E. A., \& Kodera, S. (2020). Influence of absolute humidity, temperature and population density on COVID-19 spread and decay durations: multi-prefecture study in Japan. 17(15). doi: https://doi. org/10.3390/ijerph17155354

Rendana M (2020) Impact of the wind conditions on COVID-19 pandemic: a new insight for direction of the spread of the virus. Urban Clim 34:100680. https://doi.org/10.1016/j.uclim.2020.100680

Riddell S, Goldie S, Hill A, Eagles D, Drew TW (2020) The effect of temperature on persistence of SARS-CoV-2 on common surfaces. Virol J 17(1):145. https://doi.org/10.1186/s12985-020-01418-7

Rosario DKA, Mutz YS, Bernardes PC, Conte-Junior CA (2020) Relationship between COVID-19 and weather: case study in a tropical country. Int J Hyg Environ Health 229:113587. https://doi.org/ 10.1016/j.ijheh.2020.113587

Roy MP (2020) Short report - COVID-19 and weather parameters: Pune, India. Eur Rev Med Pharmacol Sci 24(19):10203-10204. https:// doi.org/10.26355/eurrev 20201023241

Rubin D, Huang J, Fisher BT, Gasparrini A, Tam V, Song L, Wang X, Kaufman J, Fitzpatrick K, Jain A, Griffis H, Crammer K, Morris J, Tasian G (2020) Association of social distancing, population density, and temperature with the instantaneous reproduction number of SARS-CoV-2 in counties across the United States. JAMA Netw
Open 3(7):e2016099. https://doi.org/10.1001/jamanetworkopen. 2020.16099

Runkle JD, Sugg MM, Leeper RD, Rao Y, Matthews JL, Rennie JJ (2020) Short-term effects of specific humidity and temperature on COVID-19 morbidity in select US cities. Sci Total Environ 740: 140093. https://doi.org/10.1016/j.scitotenv.2020.140093

Sajadi MM, Habibzadeh P, Vintzileos A, Shokouhi S, Miralles-Wilhelm F, Amoroso A (2020) Temperature, humidity, and latitude analysis to estimate potential spread and seasonality of coronavirus disease 2019 (COVID-19). JAMA Netw Open 3(6):e2011834. https://doi. org/10.1001/jamanetworkopen.2020.11834

Sehra ST, Salciccioli JD, Wiebe DJ, Fundin S, Baker JF (2020) Maximum daily temperature, precipitation, ultra-violet light and rates of transmission of SARS-Cov-2 in the United States. Clin Infect Dis. https://doi.org/10.1093/cid/ciaa681

Singh AK, Agrawal B, Sharma A, Bera B (2020) Significant impacts of COVID-19 lockdown on urban air pollution in Kolkata (India) and amelioration of environmental health. J Public Aff 23:1-28. https:// doi.org/10.1007/s10668-020-00898-5

Sobral MFF, Duarte GB, da Penha Sobral AIG, Marinho MLM, de Souza Melo A (2020) Association between climate variables and global transmission oF SARS-CoV-2. Sci Total Environ 729:138997. https://doi.org/10.1016/j.scitotenv.2020.138997

Thorsson S, Honjo T, Lindberg F, Eliasson I, Lim E-M (2007) Thermal comfort and outdoor activity in Japanese urban public places. Environment and Behavior 39(5):660-684

timeanddate. (2020). World temperatures - weather around the world, Retrieved 10 Nov, 2020, from https://www.timeanddate.com/

Van Doremalen N, Bushmaker T, Morris DH, Holbrook MG, Gamble A, Williamson BN et al (2020) Aerosol and surface stability of SARSCoV-2 as compared with SARS-CoV-1. New England Journal of Medicine 382(16):1564-1567

Virlogeux V, Fang VJ, Wu JT, Ho L-M, Peiris JSM, Leung GM, Cowling BJ (2015) Brief report: incubation period duration and severity of clinical disease following severe acute respiratory syndrome coronavirus infection. Epidemiology (Cambridge, Mass.) 26(5):666669. https://doi.org/10.1097/EDE.0000000000000339

Watanabe T, Bartrand TA, Weir MH, Omura T, Haas CN (2010) Development of a dose-response model for SARS coronavirus. Risk Analysis 30(7):1129-1138. https://doi.org/10.1111/j.15396924.2010.01427.x

WHO. (2021). COVID-19 weekly epidemiological update. Retrieved 1 March, 2021, from https://www.who.int/emergencies/diseases/ novel-coronavirus-2019/situation-reports

Wu JT, Leung K, Leung GM (2020a) Nowcasting and forecasting the potential domestic and international spread of the 2019-nCoV outbreak originating in Wuhan, China: a modelling study. The Lancet 395(10225):689-697. https://doi.org/10.1016/S0140-6736(20) 30260-9

Wu Y, Jing W, Liu J, Ma Q, Yuan J, Wang Y, du M, Liu M (2020b) Effects of temperature and humidity on the daily new cases and new deaths of COVID-19 in 166 countries. Sci Total Environ 729: 139051. https://doi.org/10.1016/j.scitotenv.2020.139051

Xie J, Zhu Y (2020) Association between ambient temperature and COVID-19 infection in 122 cities from China. Sci Total Environ 724:138201. https://doi.org/10.1016/j.scitotenv.2020.138201

Zhao H, Che H, Zhang X, Ma Y, Wang Y, Wang H, Wang Y (2013) Characteristics of visibility and particulate matter (PM) in an urban area of Northeast China. Atmos Pollut Res 4(4):427-434. https://doi. org/10.5094/APR.2013.049

Publisher's note Springer Nature remains neutral with regard to jurisdictional claims in published maps and institutional affiliations. 